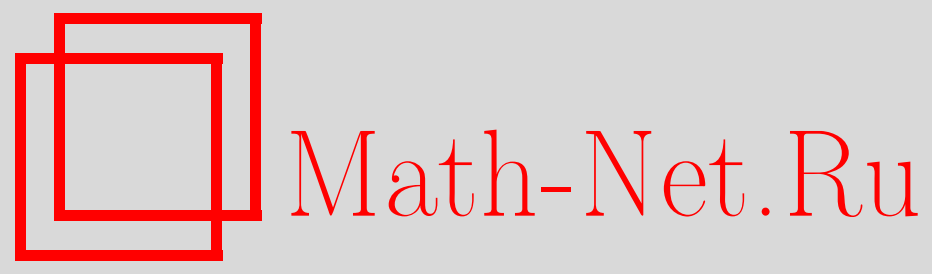

В. Л. Куракин, Полиномиальные преобразования линейных рекуррентных последовательностей над конечными коммутативными кольцами, Дискрет. матем., 2000, том 12, выпуск $3,3-36$

DOI: https://doi.org/10.4213/dm342

Использование Общероссийского математического портала Math-Net.Ru подразумевает, что вы прочитали и согласны с пользовательским соглашением http://www . mathnet.ru/rus/agreement

Параметры загрузки:

IP : 54.237 .206 .68

26 апреля 2023 г., 05:37:59 


\title{
Полиномиальные преобразования линейных рекуррентных последовательностей над конечными коммутативными кольцами
}

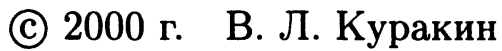

\begin{abstract}
Пусть $u$ - линейная рекуррентная последовательность (ЛРП) над конечным коммутативным локальным кольцом $R$ с единицей и $\Phi(x) \in R[x]$. В работе найден характеристический многочлен $H(x)$ и получена верхняя оценка ранга (линейной сложности) над кольцом $R$ для последовательности $v=\Phi(u)$. В случае, когда $\bar{u}-$ ЛРП максимального периода над полем вычетов $\bar{R}=R / J(R)=$ $\mathrm{GF}(q)$ кольца $R$ и $\operatorname{deg} \Phi(x) \leqslant q-1$, доказано, что эта оценка достигается и $H(x)$ является минимальным многочленом ЛРП $v$. Аналогичные результаты получены для последовательности $v=\Phi\left(u_{1}, \ldots, u_{K}\right)$, получающейся полиномиальным преобразованием нескольких линейных рекуррент $u_{1}, \ldots, u_{K}$ над кольцом $R$.
\end{abstract}

\section{1. Введение}

Пусть $R$ - коммутативное кольцо с единицей, $u$ - линейная рекуррентная последовательность (ЛРП) над кольцом $R$ с характеристическим многочленом $F(x) \in R[x]$ степени $m$. Последовательность

$$
v(i)=\Phi(u(i), \ldots, u(i+s-1)), \quad i \geqslant 0,
$$

где $\Phi\left(x_{1}, \ldots, x_{s}\right) \in R\left[x_{1}, \ldots, x_{s}\right], s \geqslant 1$, будем называть полиномиальным преобразованием последовательности $u$. Последовательность $v$ является линейной рекуррентой над кольцом $R$. Рангом (или линейной сложностью) ЛРП $v$ будем называть степень ее минимального многочлена, то есть характеристического многочлена наименьшей степени. Наша цель - описать характеристический многочлен ЛРП $v$ возможно меньшей степени и оценить ранг ЛРП $v$, а в некоторых случаях - найти минимальный многочлен и точное значение ранга ЛРП $v$.

Для линейных рекуррент над полем (в большинстве случаев конечным) эта задача широко исследовалась в $[11-19,22,24]$. При этом использовались результаты о произведении линейных рекуррент над полем (см. [25], а также $[23,8])$ и аппарат биномиального представления последовательностей над полем, частным случаем которого является представление линейных рекуррент над конечным полем функцией след.

Если $P=\mathrm{GF}(q), u-$ ЛРП над $P$ с неприводимым характеристическим многочленом степени $m, \Phi(x)=\sum_{d \geqslant 0} \varphi_{d} x^{d} \in R[x]$ и $D=\left\{d \geqslant 0: \varphi_{d} \neq 0\right\}$, то в указанных 
работах доказана, в частности, оценка

$$
\operatorname{rank} \Phi(u) \leqslant \sum_{d \in D}\left(\begin{array}{c}
m+d-1 \\
d
\end{array}\right)
$$

где через $\Phi(u)$ обозначается последовательность со знаками $v(i)=\Phi(u(i)), i \geqslant 0$. Если $q=p-$ простое число, $u$ - ЛРП максимального периода над полем $P, \operatorname{deg} \Phi \leqslant$ $p-1$, то эта оценка достигается и неравенство обращается в равенство. Если $q=p^{l}$, где $p$ простое, $l \geqslant 1$, то справедлива более точная оценка

$$
\operatorname{rank} \Phi(u) \leqslant \sum_{d \in D}\left(\begin{array}{c}
m+\nu_{0}(d)-1 \\
\nu_{0}(d)
\end{array}\right) \ldots\left(\begin{array}{c}
m+\nu_{l-1}(d)-1 \\
\nu_{l-1}(d)
\end{array}\right)
$$

где $\nu_{0}(d), \nu_{1}(d), \ldots$ - $p$-ичные разряды числа $d$. Если $u-$ ЛРП максимального периода и $\operatorname{deg} \Phi \leqslant q-1$, то неравенство (2) обращается в равенство.

Нашей целью является получение аналогичных результатов для линейных рекуррент над конечным коммутативным кольцом $R$ с единицей. Стандартными рассуждениями задача сводится к случаю локального кольца $R$, и основные результаты статьи получены в этом предположении. Ряд результатов сохраняется также для некоторых классов бесконечных колец, об этом говорится в замечаниях в конце разделов.

Перечислим основные результаты работы. Чтобы не перегружать введение, мы не формулируем результаты в том общем виде, в котором они доказываются ниже, а ограничиваемся наиболее интересными частными случаями.

Произведение последовательностей над произвольным коммутативным кольцом с единицей рассматривалось в $[20,21]$, где доказано, что

$$
\operatorname{rank} u v \leqslant \operatorname{rank} u \operatorname{rank} v \text {. }
$$

Используя этот результат, легко выводится оценка (см. следствие 1)

$$
\operatorname{rank} \Phi(u) \leqslant \sum_{d \in D} m^{d}
$$

Однако, эта оценка менее точная, чем (1), и не является ее аналогом ни по формулировке, ни по методам доказательства.

Оценка, аналогичная (1), доказывается нами в разделе 4 для ЛРП $u$ над конечным коммутативным локальным кольцом $R$ (см. теорему 2 ). В частности, если характеристический многочлен ЛРП $u$ над кольцом $R$ сепарабелен, то вид полученной оценки совпадает с (1) (см. формулу (16)). Используемые в доказательстве свойства многочленов над кольцом $R$ и результаты о биномиальном представлении линейных рекуррент над $R$ излагаются предварительно в разделе 3 .

Доказанные в разделе 4 оценки являются простыми и легко вычислимыми, однако, они не достаточно точные и не являются достижимыми для класса всех конечных коммутативных колец с единицей. Основные результаты работы получаются в разделах 5 и 6. В предположении, что характеристический многочлен ЛРП $u$ над кольцом $R$ сепарабелен, доказывается более точная оценка ранга ЛРП $\Phi(u)$ (см. теорему 5 и следствие 4). Если образ $\bar{u}$ ЛРП $u$ над полем вычетов $\bar{R}=R / J(R)=\mathrm{GF}(q)$ кольца $R$ является линейной рекуррентой максимального периода и $\operatorname{deg} \Phi(x) \leqslant q-1$, 
то эта оценка достигается. В последнем случае описан минимальный многочлен и найдено точное значение ранга ЛРП $\Phi(u)$ (теорема 7). При условии, что $R-$ кольцо главных идеалов, в частности, кольцо Галуа или примарное кольцо вычетов $Z_{p^{n}}$, описывается аннулятор последовательности $\Phi(u)$ (следствие 8). Указанная оценка является достаточно громоздкой и здесь не приводится. Она учитывает порядки коэффициентов многочлена $\Phi(x)$ в группе $(R,+)$. Если $R=\mathrm{GF}(q)$, то эта оценка превращается в (2).

Аналогичные результаты получены для полиномиальных преобразований нескольких последовательностей, то есть для последовательности

$$
v=\Phi\left(u_{1}, \ldots, u_{K}\right),
$$

где $u_{1}, \ldots, u_{K}$ - линейные рекурренты, $\Phi\left(x_{1}, \ldots, x_{K}\right)$ - многочлен над кольцом $R$. Так, в случае, когда характеристические многочлены $F_{1}(x), \ldots, F_{K}(x)$ последовательностей $u_{1}, \ldots, u_{K}$ сепарабельны и имеют степени $m_{1}, \ldots, m_{K}$ соответственно, в теореме 4 доказывается оценка

$$
\operatorname{rank} \Phi\left(u_{1}, \ldots, u_{K}\right) \leqslant \sum_{\left(d_{1}, \ldots, d_{K}\right) \in D}\left(\begin{array}{c}
m_{1}+d_{1}-1 \\
d_{1}
\end{array}\right) \ldots\left(\begin{array}{c}
m_{K}+d_{K}-1 \\
d_{K}
\end{array}\right)
$$

где $D$ - множество векторов $\left(d_{1}, \ldots, d_{K}\right) \in \mathbf{N}_{0}^{K}$ таких, что в запись многочлена $\Phi$ входит с ненулевым коэффициентом моном $x_{1}^{d_{1}} \ldots x_{K}^{d_{K}}$. Однако, эта оценка не является достижимой, в частности, не учитывает свойств кольца $R$. В разделе 5 получена более точная, но и более громоздкая, оценка (теорема 6 и следствие 5), а в разделе 6 доказывается, чго если $\bar{u}_{1}, \ldots, \bar{u}_{K}-$ ЛРП максимального периода над полем $\bar{R}$, числа $m_{1}, \ldots, m_{K}$ попарно взаимно просты и степень многочлена $\Phi\left(x_{1}, \ldots, x_{K}\right)$ по переменной $x_{k}$ меньше $M_{k}=\min \left\{q, m_{k},(q-2) m_{k} /(q-1)+1\right\}, k=1, \ldots, K$, то оценка достигается (теорема 8). В последнем случае описан минимальный многочлен и найдено точное значение ранга ЛРП $\Phi\left(u_{1}, \ldots, u_{K}\right)$. Отметим, что если $R=\mathrm{GF}(q)$ - конечное поле, то эта оценка также справедлива и обобщает результат работы [22] на более широкий класс многочленов $\Phi\left(x_{1}, \ldots, x_{K}\right)$, не свободных от квадратов переменных.

Полиномиальные преобразования линейных рекуррент над кольцом $R=Z_{p^{2}}$ исследовались автором в [7]. Результаты данной работы обобщают и расширяют результаты [7]. Для тех утверждений данной работы, которые являются аналогами соответствующих утверждений из [7], в ряде случаев найдены более простые доказательства.

\section{2. Верхние оценки ранга}

В этом разделе будут получены верхние оценки рангов полиномиальных преобразований линейных рекуррентных последовательностей над произвольным коммутативным кольцом с единицей.

Всюду далее термин кольцо означает коммутативное кольцо с единицей. Множество последовательностей над кольцом $R$ обозначим $R^{\infty}$. Произведением многочлена

$$
F(x)=\sum_{s \geqslant 0} c_{s} x^{s} \in R[x]
$$


на последовательность $u \in R^{\infty}$ называется последовательность $v=F(x) u \in R^{\infty}$ со знаками

$$
v(i)=\sum_{s \geqslant 0} c_{s} u(i+s), \quad i \geqslant 0 .
$$

Относительно этой операции абелева группа $\left(R^{\infty},+\right)$ образует левый модуль над кольцом $R[x]$ (см. [4], глава 25, теорема 1 , или $[9,19])$. Если $F(x) u=0$, то будем говорить, что многочлен $F(x)$ аннулирует последовательность $u$. Идеал

$$
\operatorname{Ann}(u)=\{F(x) \in R[x]: F(x) u=0\}
$$

кольца $R[x]$ называется аннулятором последовательности $u$. Если последовательность $u$ аннулируется некоторым унитарным многочленом $F(x)$, то она называется линейной рекуррентной последовательностью (ЛРП) над кольцом $R$, a унитарный многочлен $F(x)$ - ее характеристическим многочленом. Множество всех ЛРП над $R$ с характеристическим многочленом $F(x)$ будем обозначать $L_{R}(F)$. Характеристический многочлен ЛРП $u$ наименьшей степени называется ее минимальным многочленом, а степень минимального многочлена называется рангом $\operatorname{rank}_{R} u=\operatorname{rank} u$ ЛРП $u$. Минимальный многочлен ЛРП над полем определен однозначно, в общем случае минимальный многочлен ЛРП над кольцом определен неоднозначно.

Напомним, что сопровождающей матрицей для унитарного многочлена

$$
F(x)=x^{m}+c_{m-1} x^{m-1}+\ldots+c_{1} x+c_{0} \in R[x]
$$

называется матрица

$$
S=S(F)=\left(\begin{array}{ccccc}
0 & 0 & \ldots & 0 & -c_{0} \\
e & 0 & \ldots & 0 & -c_{1} \\
0 & e & \ldots & 0 & -c_{2} \\
& & \ldots & & \\
0 & 0 & \ldots & e & -c_{m-1}
\end{array}\right)
$$

Характеристический многочлен этой матрицы равен $F(x)$. Пусть

$$
S(F)^{\otimes d}=S(F) \otimes \ldots \otimes S(F),
$$

где тензорное произведение матриц берется $d \geqslant 1$ раз, и $S(F)^{\otimes 0}=(e)$, где $(e)-$ единичная матрица размера $1 \times 1$. Тогда $S(F)^{\otimes d}$ - матрица размера $m^{d} \times m^{d}, d \geqslant 0$. Результаты этого раздела основаны на следующей теореме из [20].

Теорема 1 ([20]). Если $u, v$ - ЛРП над кольцом $R$ с характеристическими многочленами $F(x), G(x) \in R[x]$ степеней $m, n \geqslant 1$, то характеристический многоилен матрицы $S(F) \otimes S(G)$ является характеристическим многочленом ЛРП $и$, $u$ rank $u v \leqslant m n$.

Предложение 1. Пусть $u-Л P \Pi$ над кольчом $R$ с характеристическим многоиленом $F(x) \in R[x]$ степени $m \geqslant 1, \Phi\left(x_{1}, \ldots, x_{s}\right) \in R\left[x_{1}, \ldots, x_{s}\right]$,

$$
v(i)=\Phi(u(i), \ldots, u(i+s-1)), \quad i \geqslant 0,
$$

$D$ - множество чисел $d \geqslant 0$ тахих, что в запись многочлена $\Phi$ входит $c$ ненулевьм хоэффичиентом моном степени $d, F_{d}(x)$ - харахтеристический многочлен матрици $S(F)^{\otimes d}$. 
Тогда многочлен

$$
H(x)=\prod_{d \in D} F_{d}(x)
$$

является харахтеристическим многочленом ЛРП $v, u$

$$
\operatorname{rank} v \leqslant \Phi^{*}(m)
$$

əde

$$
\Phi^{*}(x)=\sum_{d \in D} x^{d} \in Z[x]
$$

Дохазательство. Многочлен $\Phi$ можно записать в виде

$$
\Phi=\sum_{d \in D} \Phi_{d}
$$

где $\Phi_{d}\left(x_{1}, \ldots, x_{s}\right)$ - форма степени $d$. Пусть

$$
v_{d}(i)=\Phi_{d}(u(i), \ldots, u(i+s-1)), \quad i \geqslant 0 .
$$

Тогда

$$
v=\sum_{d \in D} v_{d}
$$

По теореме 1 многочлен $F_{d}(x)$ является характеристическим многочленом ЛРП $v_{d}$, $d \geqslant 2$; при $d=0$ и $d=1$ это утверждение, очевидно, также выполнено. Следовательно, многочлен $H(x)$ является характеристическим многочленом ЛРП $v$. Так как $\operatorname{deg} F_{d}(x)=m^{d}, d \geqslant 0$, то отсюда вытекает оценка (3).

Следствие 1. Пусть $u-$ ЛРП над холъцом $R$ с харажтеристическим многочленом $F(x) \in R[x]$ степени $m, \Phi(x) \in R[x]$. Тогда

$$
\operatorname{rank} \Phi(u) \leqslant \Phi^{*}(m),
$$

где $\Phi^{*}(x)$ - многочлен над кольцом целых иисел, полученный из $\Phi(x)$ заменой всех ненулевых коэфичиентов на 1.

Аналогично доказывается следующий результат о полиномиальных преобразованиях нескольких последовательностей.

Предложение 2. Пусть $u_{1}, \ldots, u_{K}$ - линейнье рехурренты над хольчом $R$ с характеристическими многочленами $F_{1}(x), \ldots, F_{K}(x) \in R[x]$, соответственно, степеней $m_{1}, \ldots, m_{K} \geqslant 1, \Phi\left(x_{1,1}, \ldots, x_{1, s_{1}}, \ldots, x_{K, 1}, \ldots, x_{K, s_{K}}\right)$ - многочлен над кольчом $R$, где $s_{1}, \ldots, s_{K} \geqslant 0$,

$$
v(i)=\Phi\left(u_{1}(i), \ldots, u_{1}\left(i+s_{1}-1\right), \ldots, u_{K}(i), \ldots, u_{K}\left(i+s_{K}-1\right)\right), \quad i \geqslant 0,
$$

$D$ - множество векторов $\left(d_{1}, \ldots, d_{K}\right) \in \mathbf{N}_{0}^{K}$ тахих, что в записв многочлена $\Phi$ входит с ненулевъм коэффичиентом моном, сумма степеней хоторого по переменным $x_{k, 1}, \ldots, x_{k, s_{k}}$ равна $d_{k}, k=1, \ldots, K, F_{\left(d_{1}, \ldots, d_{K}\right)}(x)$ - характеристический многочлен матричь $S\left(F_{1}\right)^{\otimes d_{1}} \otimes \ldots \otimes S\left(F_{K}\right)^{\otimes d_{K}}$. 
Тогда многочлен

$$
H(x)=\prod_{\left(d_{1}, \ldots, d_{K}\right) \in D} F_{\left(d_{1}, \ldots, d_{K}\right)}(x)
$$

является характеристическим многочленом ЛРП $v, u$

$$
\operatorname{rank} v \leqslant \Phi^{*}\left(m_{1}, \ldots, m_{K}\right),
$$

əде

$$
\Phi^{*}\left(x_{1}, \ldots, x_{K}\right)=\sum_{\left(d_{1}, \ldots, d_{K}\right) \in D} x_{1}^{d_{1}} \ldots x_{K}^{d_{K}} \in Z\left[x_{1}, \ldots, x_{K}\right] .
$$

Следствие 2. Пусть в условиях предложения $2 s_{1}=\ldots=s_{K}=1$, то есть $v=$ $\Phi\left(u_{1}, \ldots, u_{K}\right)$. Тогда

$$
\operatorname{rank} \Phi\left(u_{1}, \ldots, u_{K}\right) \leqslant \Phi^{*}\left(m_{1}, \ldots, m_{K}\right)
$$

где $\Phi^{*}\left(x_{1}, \ldots, x_{K}\right)$ - многочлен над кольцом цельх чисел, полученнъй из $\Phi\left(x_{1}, \ldots, x_{K}\right)$ заменой всех ненулевых коэффичиентов на 1.

Оценки (3) и (4) универсальны: они верны для линейных рекуррент с любыми характеристическими многочленами над произвольным кольцом $R$. Однако для линейных рекуррент над конечными кольцами (на самом деле и для более широкого класса колец, см. замечания 1, 2 и 4) можно доказать более точные оценки. Это делается в следующих разделах.

Пусть кольцо $R$ представляется в виде прямой суммы идеалов

$$
R=R_{1}+\ldots+R_{t}
$$

и соответственно единица кольца $R$ представляется в виде

$$
e=e_{1}+\ldots+e_{t}, \quad e_{j} \in R_{j}, \quad j=1, \ldots, t .
$$

Тогда $R_{j}=e_{j} R$ - кольцо с единицей $e_{j}, j=1, \ldots, t$, и каждый многочлен $F(x) \in R[x]$ и каждая последовательность $u \in R^{\infty}$ однозначно представляются в виде

$$
\begin{aligned}
F(x) & =F_{1}(x)+\ldots+F_{t}(x), \quad F_{j}(x)=e_{j} F(x) \in R_{j}[x] \\
u & =u_{1}+\ldots+u_{t}, \quad u_{j}=e_{j} u \in R_{j}^{\infty}, \quad j=1, \ldots, t
\end{aligned}
$$

При этом, если многочлен $F(x)$ унитарен и является характеристическим многочленом ЛРП $u$, то каждый из многочленов $F_{j}(x)$ унитарен в кольце $R_{j}[x]$ и является характеристическим многочленом ЛРП $u_{j}$ при $j=1, \ldots, t$. Обратно, если $F_{j}(x) \in R_{j}[x]$ - характеристический многочлен ЛРП $u_{j}$ при $j=1, \ldots, t$, то многочлен

$$
F(x)=\sum_{j=1}^{t} x^{m-m_{j}} F_{j}(x), \quad m=\max \left\{m_{1}, \ldots, m_{t}\right\}, \quad m_{j}=\operatorname{deg} F_{j}(x), \quad j=1, \ldots, t .
$$

является характеристическим многочленом ЛРП $u$. При этом

$$
\operatorname{rank}_{R} u=\max \left\{\operatorname{rank}_{R_{1}} u_{1}, \ldots, \operatorname{rank}_{R_{t}} u_{t}\right\}
$$


Таким образом, задача нахождения характеристического многочлена и ранга ЛРП $u$ над кольцом $R$ сводится к задаче нахождения характеристических многочленов и рангов последовательностей $u_{j}$ над кольцом $R_{j}$ при $j=1, \ldots, t$.

Поскольку, согласно $[1,5]$, каждое конечное (каждое артиново) кольцо $R$ однозначно, с точностью до перестановки слагаемых, представляется в виде прямой суммы локальных конечных (артиновых) колец, то в дальнейшем мы будем предполагать, что кольцо $R$ локально.

\section{3. Необходимые сведения о конечных локальных кольцах и линейных рекуррентах}

Всюду далее, если не оговорено противное, $R$ - конечное локальное кольцо. Пусть $J(R)$ - максимальный идеал кольца $R$, совпадающий с его радикалом Джекобсона, $\bar{R}=R / J(R)=\mathrm{GF}(q)$ - поле вычетов кольца $R, n=\operatorname{ind} J(R)$ - индекс нильпотентности идеала $J(R)$, то есть наименьшее число $n \geqslant 0$ такое, что $J(R)^{n}=0$. Обозначим через $\bar{a}$ образ элемента $a \in R$ при действии естественного эпиморфизма $R \rightarrow \bar{R}$. Аналогично определяются образы $\bar{F}(x), \bar{u}$ многочлена $F(x) \in R[x]$ и последовательности $u \in R^{\infty}$.

Многочлены $F(x), G(x) \in R[x]$ будем называть взаимно простыми (над кольцом $R$ ) и писать

$$
(F(x), G(x))=e
$$

если

$$
U(x) F(x)+V(x) G(x)=e
$$

для некоторых $U(x), V(x) \in R[x]$.

Предложение 3. Если $F(x), G_{0}(x), H_{0}(x)$ - унитарные многочлены над колъцом $R$ такие, что $\bar{F}(x)=\bar{G}_{0}(x) \bar{H}_{0}(x),\left(\bar{G}_{0}(x), \bar{H}_{0}(x)\right)=\bar{e}$, то существует единственная пара унитарных многочленов $G(x), H(x) \in R[x]$ тахих, что

$$
F(x)=G(x) H(x), \quad \bar{G}(x)=\bar{G}_{0}(x), \quad \bar{H}(x)=\bar{H}_{0}(x)
$$

(лемма Гензеля);

$$
(\bar{F}(x), \bar{G}(x))=\bar{e} \Leftrightarrow(F(x), G(x))=e .
$$

Доказательство первого утверждения см. в [2, стр. 275], или в [5, стр. 323]. Второе утверждение доказано в [9, стр. 209].

Унитарный многочлен $F(x) \in R[x]$ называется примарным, если $\bar{F}(x)=\bar{G}(x)^{k}$, где $\bar{G}(x) \in \bar{R}[x]-$ неприводимый над полем $\bar{R}$ многочлен, $k \geqslant 1$.

Следствие 3. Любой унитарнъй многочлен $F(x) \in R[x]$ однозначно, $c$ точностъю до перестановки сомножителей, представляется в виде произведения примарных попарно взаимно простых многочленов:

$$
F(x)=F_{1}(x) \ldots F_{T}(x), \quad \bar{F}_{t}(x)=\bar{G}_{t}(x)^{a_{t}}, \quad a_{t} \geqslant 1, \quad t=1, \ldots, T,
$$

где многочлен $G_{t}(x) \in R[x]$ унитарен $u \bar{G}_{t}(x)$ неприводим над полем $\bar{R}$. 
Разложение (5) будем называть примарным каноническим разложением многочлена $F(x)$. При условии (5) положим

$$
a(F)=a(\bar{F})=\max \left\{a_{1}, \ldots, a_{T}\right\} .
$$

Многочлен $F(x)$ будем называть сепарабельным, если многочлен $\bar{F}(x)$ над полем $\bar{R}$ сепарабелен, то есть не имеет кратных корней в поле разложения. Поскольку конечное поле $\bar{R}$ является совершенным [3], это равносильно тому, что многочлен $\bar{F}(x)$ не имеет кратных множителей в каноническом разложении над полем $\bar{R}$, то есть условию $a(F)=1$.

Радикалом $\operatorname{rad} f(x)$ ненулевого многочлена $f(x)$ над конечным (или совершенным) полем называется унитарный многочлен, равный произведению всех неприводимых многочленов, входящих в каноническое разложение многочлена $f(x)$. Радикал многочлена над конечным полем является сепарабельным многочленом и вычисляется по формуле $\operatorname{rad} f(x)=f(x) /\left(f(x), f^{\prime}(x)\right)$.

Определение 1. Радикалом $\operatorname{rad} F(x)$ унитарного многочлена $F(x)$ над кольцом $R$ будем называть сам многочлен $F(x)$, если $F(x)$ сепарабелен, или произвольный унитарный многочлен $G(x) \in R[x]$ такой, что $\bar{G}(x)=\operatorname{rad} \bar{F}(x)$, если $F(x)$ не сепарабелен.

Радикал $G(x)=\operatorname{rad} F(x)$ многочлена $F(x)$ над кольцом $R$ является сепарабельным многочленом, удовлетворяющим условию $\bar{G}(x) \mid \bar{F}(x)$, однако не всегда $G(x) \mid F(x)$. В связи с этим полезным оказывается следующий результат.

Предложение 4. Пусть $F(x), G(x) \in R[x], \bar{G}(x) \mid \bar{F}(x)$. Тогда $G(x) \mid F(x)^{n}$, где $n=\operatorname{ind} J(R)$.

$B$ частности, $\operatorname{rad} F(x)\left|F(x)^{n} u F(x)\right|(\operatorname{rad} F)^{n a(F)}$.

Доказательство. Пусть $\bar{F}(x)=\bar{G}(x) \bar{Q}(X)$. Тогда

$$
F(x)=G(x) Q(x)+\Delta(x),
$$

где $\Delta(x) \in J(R)[x], \Delta(x)^{n}=0$. Следовательно,

$$
F^{n}=(G Q+\Delta)^{n}=\sum_{i=0}^{n}\left(\begin{array}{c}
n \\
i
\end{array}\right)(G Q)^{i} \Delta^{n-i}=\sum_{i=1}^{n}\left(\begin{array}{c}
n \\
i
\end{array}\right)(G Q)^{i} \Delta^{n-i}
$$

откуда получаем, что $G(x) \mid F(x)^{n}$. Последние два соотношения теперь следуют из того, что $\overline{(\operatorname{rad} F)}=\operatorname{rad} \bar{F} \mid \bar{F}$ и $\bar{F} \mid(\operatorname{rad} \bar{F})^{a(F)}$.

Унитарный многочлен $F(x) \in R[x]$ называется абсолютно неприводимым или многочленом Галуа, если $\bar{F}(x)$ - неприводимый многочлен над полем $\bar{R}$. Пусть $F(x)$ - многочлен Галуа степени $m$ над $R$. Тогда факторкольцо $S=R[x] / F(x)$ является локальным конечным кольцом. Можно считать, что $R \subseteq S$. Тогда $S$ называется расширением Галуа степени $m$ кольца $R$. Кольцо $S$, полученное конечным числом таких расширений, также будем называть расширением Галуа кольца $R$, а его степень $m$ считать равной степени $[\bar{S}: \bar{R}]$ расширения поля $\bar{S}=S / J(S)$ над $\bar{R}$. Кольцо $S$ является свободным $R$-модулем ранга $m$, в частности, $|S|=|R|^{m}$. Справедливы соотношения $J(S)=J(R) S$, ind $J(S)=$ ind $J(R), \bar{S}=\mathrm{GF}\left(q^{m}\right)$.

Пусть $F(x) \in R[x]$ - унитарный многочлен с каноническим разложением (5), $G(x)=\operatorname{rad} F(x), S-$ расширение Галуа кольца $R$ степени $\left[\operatorname{deg} G_{1}, \ldots, \operatorname{deg} G_{T}\right]$. Тогда 
многочлены $\bar{G}_{1}(x), \ldots, \bar{G}_{T}(x)$ и, следовательно, многочлен $\bar{G}(x)=\bar{G}_{1}(x) \ldots \bar{G}_{T}(x)$, раскладываются на линейные множители над полем $\bar{S}$. Так как многочлен $G(x)$ сепарабелен, по лемме Гензеля он однозначно раскладывается в произведение линейных множителей над $S$ :

$$
G(x)=\left(x-\vartheta_{1}\right) \ldots\left(x-\vartheta_{m}\right), \quad \vartheta_{1}, \ldots, \vartheta_{m} \in S, \quad m=\operatorname{deg} G(x) .
$$

Напомним, что биномиальной последовательностью над кольцом $S$ порядка $l \geqslant 0$ с корнем $\vartheta \in S$ называется последовательность $\vartheta^{[l]} \in S^{\infty}$, определяемая соотношениями

$$
\left(\vartheta^{[l]}(0), \ldots, \vartheta^{[l]}(l)\right)=(0, \ldots, 0, e), \quad \vartheta^{[l]}(i)=\left(\begin{array}{l}
i \\
l
\end{array}\right) \vartheta^{i-l}, \quad i>l
$$

Мы кратко пишем $\vartheta^{[l]}(i)=\left(\begin{array}{l}i \\ l\end{array}\right) \vartheta^{i-l}$ при всех $i \geqslant 0$, полагая, что если $\left(\begin{array}{l}i \\ l\end{array}\right)=0$, то и $\left(\begin{array}{l}i \\ l\end{array}\right) \vartheta^{i-l}=0$ независимо от того, определена ли степень $\vartheta^{i-l}$. Последовательность $\vartheta^{[l]}$ имеет минимальный многочлен $(x-\vartheta)^{l+1}$ и является импульсной последовательностью семейства $L_{S}\left((x-\vartheta)^{l+1}\right)$ (см. [19]).

Предложение 5. Пусть $F(x) \in R[x]-$ унитарный многочлен, $a=a(F), G(x)=$ $\operatorname{rad} F(x), m=\operatorname{deg} G(x)$ и над некоторим расширением Галуа $S$ кольца $R$ выполняется соотношение (7). Тогда произвольная ЛРП $u \in L_{R}(F)$ представляется в виде

$$
u=\sum_{r=1}^{m} \sum_{l=0}^{a n-1} c_{r l} \vartheta_{r}^{[l]}
$$

mo ecmb

$$
u(i)=\sum_{r=1}^{m} \sum_{l=0}^{a n-1} c_{r l}\left(\begin{array}{l}
i \\
l
\end{array}\right) \vartheta_{r}^{i-l}, \quad i \geqslant 0,
$$

где коэффициенты $c_{r l} \in S$ определены однозначно, $n=$ ind $J(R)$.

Если многочлен $F(x)$ сепарабелен, то $G(x)=F(x)$ и произволъная ЛРП $u \in$ $L_{R}(F)$ представляется в виде

$$
u=\sum_{r=1}^{m} c_{r} \vartheta_{r}^{[0]}
$$

mo ecms

$$
u(i)=\sum_{r=1}^{m} c_{r} \vartheta_{r}^{i}, \quad i \geqslant 0,
$$

где коэффичиенты $c_{r} \in S$ определены однозначно.

Доказательство. В силу (7) и предложения 4

$$
F(x) \mid G(x)^{a n}=\left(x-\vartheta_{1}\right)^{a n} \ldots\left(x-\vartheta_{m}\right)^{a n} .
$$

Поэтому

$$
u \in L_{R}(F) \subseteq L_{S}(F) \subseteq L_{S}\left(\left(x-\vartheta_{1}\right)^{a n} \ldots\left(x-\vartheta_{m}\right)^{a n}\right)
$$


Так как элементы $\bar{\vartheta}_{1}, \ldots, \bar{\vartheta}_{m}$ в (7) попарно различны, ввиду предложения 3 многочлены $\left(x-\vartheta_{1}\right)^{a n}, \ldots,\left(x-\vartheta_{m}\right)^{a n}$ попарно взаимно просты. Следовательно, в силу предложения 6(a) из [9]

$$
L_{S}\left(\left(x-\vartheta_{1}\right)^{a n} \ldots\left(x-\vartheta_{m}\right)^{a n}\right)=L_{S}\left(\left(x-\vartheta_{1}\right)^{a n}\right) \dot{+} \ldots \dot{+} L_{S}\left(\left(x-\vartheta_{m}\right)^{a n}\right) .
$$

Биномиальные последовательности $\vartheta^{[0]}, \ldots, \vartheta^{[a n-1]}$ принадлежат $L_{S}\left((x-\vartheta)^{a n}\right)$ и, как следует непосредственно из (8), образуют базис этого семейства как модуля над $S$. Отсюда вытекает существование и единственность представления (9) ЛРП $u$.

Если $F(x)$ сепарабелен, то $G(x)=F(x)$ и

$$
u \in L_{R}(F) \subseteq L_{S}(F)=L_{S}\left(x-\vartheta_{1}\right) \dot{+} \ldots \dot{+} L_{S}\left(x-\vartheta_{m}\right)
$$

Отсюда вытекает существование и единственность представления (10).

Соотношения (9), (10) будем называть биномиальным представлением ЛРП $u$. В дальнейшем нам потребуется следующая лемма.

Лемма 1. Пусть $\vartheta_{1}, \ldots, \vartheta_{k} \in R, l_{1}, \ldots, l_{k} \geqslant 0, i_{1}, \ldots, i_{k} \geqslant 0, k \geqslant 1$. Тогда последовательность

$$
z(i)=\vartheta_{1}^{\left[l_{1}\right]}\left(i+i_{1}\right) \ldots \vartheta_{k}^{\left[l_{k}\right]}\left(i+i_{k}\right), \quad i \geqslant 0
$$

аннулируется многочленом $\left(x-\vartheta_{1} \ldots \vartheta_{k}\right)^{l_{1}+\ldots+l_{k}+1}$.

Доказательство. Будем считать, что $\vartheta^{[l]}=0$ при $l<0$. Нетрудно видеть, что

$$
\begin{aligned}
\left(\left(x-\vartheta_{1} \ldots \vartheta_{k}\right) z\right)(i) & =z(i+1)-\vartheta_{1} \ldots \vartheta_{k} z(i) \\
& =\prod_{s=1}^{k}\left(\begin{array}{c}
i+i_{s}+1 \\
l_{s}
\end{array}\right) \vartheta_{s}^{i+i_{s}+1-l_{s}}-\vartheta_{1} \ldots \vartheta_{k} \prod_{s=1}^{k}\left(\begin{array}{c}
i+i_{s} \\
l_{s}
\end{array}\right) \vartheta_{s}^{i+i_{s}-l_{s}} \\
& =\prod_{s=1}^{k}\left(\left(\begin{array}{c}
i+i_{s} \\
l_{s}
\end{array}\right)+\left(\begin{array}{c}
i+i_{s} \\
l_{s}-1
\end{array}\right)\right) \vartheta_{s}^{i+i_{s}+1-l_{s}}-\prod_{s=1}^{k}\left(\begin{array}{c}
i+i_{s} \\
l_{s}
\end{array}\right) \vartheta_{s}^{i+i_{s}+1-l_{s}} \\
& =\sum_{A}\left(\begin{array}{c}
i+i_{1} \\
l_{1}-\alpha_{1}
\end{array}\right) \ldots\left(\begin{array}{c}
i+i_{k} \\
l_{k}-\alpha_{k}
\end{array}\right) \vartheta_{1}^{i+i_{1}+1-l_{1}} \ldots \vartheta_{k}^{i+i_{k}+1-l_{k}} \\
& =\sum_{A} \vartheta_{1}^{1-\alpha_{1}} \ldots \vartheta_{k}^{1-\alpha_{k}} \vartheta_{1}^{\left[l_{1}-\alpha_{1}\right]}\left(i+i_{1}\right) \ldots \vartheta_{k}^{\left[l_{k}-\alpha_{k}\right]}\left(i+i_{k}\right)
\end{aligned}
$$

где суммирование в двух последних суммах проводится по множеству

$$
A=\left\{\alpha_{1}, \ldots, \alpha_{k} \in\{0,1\}:\left(\alpha_{1}, \ldots, \alpha_{k}\right) \neq(0, \ldots, 0)\right\}
$$

Таким образом, последовательность $\left(x-\vartheta_{1} \ldots \vartheta_{k}\right) z$ есть линейная комбинация последовательностей

$$
\vartheta_{1}^{\left[t_{1}\right]}\left(i+i_{1}\right) \ldots \vartheta_{k}^{\left[t_{k}\right]}\left(i+i_{k}\right)
$$

таких, что

$$
t_{1}+\ldots+t_{k}=l_{1}-\alpha_{1}+\ldots+l_{k}-\alpha_{k} \leqslant l_{1}+\ldots+l_{k}-1,
$$

где $t_{1}, \ldots, t_{k} \in Z$. Индукцией по $l$ доказывается, что последовательность $\left(x-\vartheta_{1} \ldots \vartheta_{k}\right)^{l} z$ есть линейная комбинация последовательностей

$$
\vartheta_{1}^{\left[t_{1}\right]}\left(i+i_{1}\right) \ldots \vartheta_{k}^{\left[t_{k}\right]}\left(i+i_{k}\right), \quad t_{1}+\ldots+t_{k} \leqslant l_{1}+\ldots+l_{k}-l, \quad t_{1}, \ldots, t_{k} \in Z
$$


При $l=l_{1}+\ldots+l_{k}+1$ получим, что $t_{1}+\ldots+t_{k} \leqslant-1$, поэтому

$$
\vartheta_{1}^{\left[t_{1}\right]}\left(i+i_{1}\right) \ldots \vartheta_{k}^{\left[t_{k}\right]}\left(i+i_{k}\right)=0
$$

при всех наборах $t_{1}, \ldots, t_{k}$ и, следовательно, $\left(x-\vartheta_{1} \ldots \vartheta_{k}\right)^{l} z=0$.

\section{4. Верхние оценки ранга}

Пусть $u$ - ЛРП над конечным локальным кольцом $R$ с характеристическим многочленом $F(x) \in R[x]$, имеющим примарное каноническое разложение (5). Пусть

$$
a=a(F), \quad G(x)=\operatorname{rad} F(x), \quad m=\operatorname{deg} G(x),
$$

и $S$ - расширение Галуа кольца $R$, над которым многочлен $G(x)$ имеет разложение (7) на линейные множители.

При $d \geqslant 0$ пусть

$$
G^{(d)}(x)=\prod\left(x-\vartheta_{i_{1}} \ldots \vartheta_{i_{d}}\right)
$$

где произведение берется по всем различным элементам

$$
\vartheta_{i_{1}} \ldots \vartheta_{i_{d}}, \quad 1 \leqslant i_{1} \leqslant \ldots \leqslant i_{d} \leqslant m
$$

кольца $S$. В частности,

$$
G^{(0)}(x)=x-e, \quad G^{(1)}(x)=G(x) .
$$

Так как многочлен $G^{(d)}(x)$ не изменится при произвольной перестановке элементов $\vartheta_{1}, \ldots, \vartheta_{m}$, коэффициенты многочлена $G^{(d)}(x)$ являются симметрическими функциями элементов $\vartheta_{1}, \ldots, \vartheta_{m}$. По основной теореме о симметрических многочленах коэффициенты многочлена $G^{(d)}(x)$ полиномиально выражаются через элементарные симметрические многочлены от $\vartheta_{1}, \ldots, \vartheta_{m}$, то есть, ввиду (7), через коэффициенты многочлена $G(x)$. Следовательно, $G^{(d)}(x) \in R[x]$. Многочлен $G^{(d)}(x)$ также можно записать в виде

$$
G^{(d)}(x)=\prod\left(x-\vartheta_{1}^{j_{1}} \ldots \vartheta_{m}^{j_{m}}\right)
$$

где произведение берется по всем различным элементам

$$
\vartheta_{1}^{j_{1}} \ldots \vartheta_{m}^{j_{m}}, \quad j_{1}+\ldots+j_{m}=d, \quad j_{1}, \ldots, j_{m} \geqslant 0
$$

кольца $S$. Степень многочлена $G^{(d)}(x)$ не превосходит числа решений уравнения $j_{1}+\ldots+j_{m}=d$ в целых неотрицательных числах $j_{1}, \ldots, j_{m}$. Следовательно,

$$
\operatorname{deg} G^{(d)}(x) \leqslant\left(\begin{array}{c}
m+d-1 \\
d
\end{array}\right), \quad d \geqslant 0 .
$$

Предложение 6. Для фиксированных $i_{1}, \ldots, i_{d} \geqslant 0, d \geqslant 1$ пусть

$$
w(i)=u\left(i+i_{1}\right) \ldots u\left(i+i_{d}\right), \quad i \geqslant 0 .
$$


Тогда многочлен $G^{(d)}(x)^{(a n-1) d+1}$ являетсл характеристическим многочленом ЛРП $w, u$

$$
\operatorname{rank} w \leqslant\left(\begin{array}{c}
m+d-1 \\
d
\end{array}\right)((a n-1) d+1) .
$$

Если многочлен $F(x)$ сепарабелен, то $G(x)=F(x), m=\operatorname{deg} F(x)$, многочлен $G^{(d)}(x)$ является характеристическим многочленом ЛРП $w, u$

$$
\operatorname{rank} w \leqslant\left(\begin{array}{c}
m+d-1 \\
d
\end{array}\right) .
$$

Дожазательство. По предложению 5 ЛРП $и$ имеет биномиальное представление (9). Тогда

$$
\begin{aligned}
w(i) & =u\left(i+i_{1}\right) \ldots u\left(i+i_{d}\right)=\prod_{j=1}^{d}\left(\sum_{r=1}^{m} \sum_{l=0}^{a n-1} c_{r l} \vartheta_{r}^{[l]}\left(i+i_{j}\right)\right) \\
& =\sum_{A(m, d)} c_{r_{1}, l_{1}} \ldots c_{r_{d}, l_{d}} \vartheta_{r_{1}}^{\left[l_{1}\right]}\left(i+i_{1}\right) \ldots \vartheta_{r_{d}}^{\left[l_{d}\right]}\left(i+i_{d}\right), \quad i \geqslant 0
\end{aligned}
$$

где суммирование в последней сумме проводится по множеству

$$
A(m, d)=\left\{\left(r_{j}, l_{j}\right): 1 \leqslant r_{j} \leqslant m, 0 \leqslant l_{j} \leqslant a n-1, j=1, \ldots, d\right\} .
$$

По лемме 1 последовательность

$$
z(i)=\vartheta_{r_{1}}^{\left[l_{1}\right]}\left(i+i_{1}\right) \ldots \vartheta_{r_{d}}^{\left[l_{d}\right]}\left(i+i_{d}\right), \quad i \geqslant 0
$$

аннулируется многочленом $\left(x-\vartheta_{r_{1}} \ldots \vartheta_{r_{d}}\right)^{l_{1}+\ldots+l_{d}+1}$. Так как $0 \leqslant l_{j} \leqslant a n-1$ при $j=1, \ldots, d$, то

$$
l_{1}+\ldots+l_{d}+1 \leqslant(a n-1) d+1,
$$

и указанный многочлен делит $G^{(d)}(x)^{(a n-1) d+1}$ в силу определения (11). Поэтому последовательность $z$ аннулируется многочленом $G^{(d)}(x)^{(a n-1) d+1}$. Теперь из (14) следует, что и ЛРП $w$ аннулируется многочленом $G^{(d)}(x)^{(a n-1) d+1}$. Оценка ранга ЛРП $w$ следует из (13).

Если многочлен $F(x)$ сепарабелен, то ЛРП $u$ имеет биномиальное представление (10) и

$$
\begin{aligned}
w(i) & =u\left(i+i_{1}\right) \ldots u\left(i+i_{d}\right)=\prod_{j=1}^{d}\left(\sum_{r=1}^{m} c_{r} \vartheta_{r}^{i+i_{j}}\right) \\
& =\sum_{1 \leqslant r_{1}, \ldots, r_{d} \leqslant m} c_{r_{1}} \ldots c_{r_{d}} \vartheta_{r_{1}}^{i_{1}} \ldots \vartheta_{r_{d}}^{i_{d}}\left(\vartheta_{r_{1}} \ldots \vartheta_{r_{d}}\right)^{i}, \quad i \geqslant 0 .
\end{aligned}
$$

Так как каждая последовательность $\left(\vartheta_{r_{1}} \ldots \vartheta_{r_{d}}\right)^{i}, i \geqslant 0$, аннулируется многочленом $G^{(d)}(x)$, то и последовательность $w$ аннулируется многочленом $G^{(d)}(x)$.

Теорема 2. Пусть $u-Л Р П$ над кольцом $R$ с характеристическим многочленом $F(x) \in R[x]$ степени $m \geqslant 1, n=$ ind $J(R), a=a(F), G(x)=\operatorname{rad} F(x), m=\operatorname{deg} G(x)$, $\Phi\left(x_{1}, \ldots, x_{s}\right) \in R\left[x_{1}, \ldots, x_{s}\right], r=\operatorname{deg} \Phi$,

$$
v(i)=\Phi(u(i), \ldots, u(i+s-1)), \quad i \geqslant 0,
$$


$D$ - множество чисел $d \geqslant 0$ таких, что в запись многочлена $\Phi$ входит с ненулевим коэффициентом моном степени d. Тогда многочлен

$$
H(x)=\prod_{d \in D} G^{(d)}(x)^{(a n-1) d+1}
$$

является характеристическим многочленом ЛРП $v, u$

$$
\operatorname{rank} v \leqslant \sum_{d \in D}\left(\begin{array}{c}
m+d-1 \\
d
\end{array}\right)((a n-1) d+1) \leqslant\left(\begin{array}{c}
m+r \\
r
\end{array}\right)((a n-1) r+1) .
$$

Если многочлен $F(x)$ сепарабелен, то $G(x)=F(x), m=\operatorname{deg} F(x)$, многочлен

$$
H_{1}(x)=\prod_{d \in D} G^{(d)}(x)
$$

является характеристическим многочленом ЛРП $v, u$

$$
\operatorname{rank} v \leqslant \sum_{d \in D}\left(\begin{array}{c}
m+d-1 \\
d
\end{array}\right) \leqslant\left(\begin{array}{c}
m+r \\
r
\end{array}\right)
$$

Доказательство. Последовательность $v$ есть линейная комбинация последовательностей

$$
w(i)=u\left(i+i_{1}\right) \ldots u\left(i+i_{d}\right), \quad 0 \leqslant i_{1}, \ldots, i_{d} \leqslant s-1, \quad d \in D,
$$

с некоторыми коэффициентами, равными коэффициентам многочлена $\Phi$ (при $d=0$ получаем последовательность-константу $\Phi(0, \ldots, 0))$. Ввиду предложения 6 последовательность $w$ аннулируется многочленом $G^{(d)}(x)^{(a n-1) d+1}$ (или $G^{(d)}(x)$, если $F(x)$ сепарабелен). Следовательно, последовательность $v$ аннулируется многочленом $H(x)$ (многочленом $H_{1}(x)$, если $F(x)$ сепарабелен). Оценки ранга следуют из (13) и соотношения (см., например, [10])

$$
\sum_{d=0}^{r}\left(\begin{array}{c}
m+d-1 \\
d
\end{array}\right)=\left(\begin{array}{c}
m+r \\
r
\end{array}\right)
$$

Отметим, что если многочлен $F(x)$ сепарабелен, то оценка (16) является более точной, чем $(3)$, поскольку $\left(\begin{array}{c}m+d-1 \\ d\end{array}\right) \leqslant m^{d}$. Если многочлен $F(x)$ не сепарабелен, то более точной может оказаться как оценка (3), так и оценка (15). Например, если $\Phi(x)=x^{d}$ и в разложении (7) $a_{1}=\ldots=a_{T}=a$, то $\operatorname{deg} F(x)=a m$, и правые части оценок (3), (15) имеют вид

$$
(a m)^{d}, \quad\left(\begin{array}{c}
m+d-1 \\
d
\end{array}\right)((a n-1) d+1) .
$$

Какая из оценок лучше, зависит от параметров $a, m, n$ и $d$.

Рассмотрим теперь полиномиальные преобразования нескольких последовательностей. Пусть $F_{1}(x), \ldots, F_{K}(x)$ - унитарные многочлены над кольцом $R$, имеющие примарные канонические разложения

$$
\begin{aligned}
F_{k}(x) & =F_{k, 1}(x) \ldots F_{k, T_{k}}(x), \\
\bar{F}_{k t}(x) & =\bar{G}_{k t}(x)^{a_{k t}}, \quad a_{k t} \geqslant 1, \quad t=1, \ldots, T_{k}, \quad k=1, \ldots, K .
\end{aligned}
$$


Введем обозначения

$$
G_{k}(x)=\operatorname{rad} F_{k}(x), G(x)=\operatorname{rad} G_{1}(x) \ldots G_{K}(x)=\operatorname{rad} F_{1}(x) \ldots F_{K}(x), m=\operatorname{deg} G(x) .
$$

Многочлен $G(x)$ является сепарабельным. Пусть $S$ - расширение Галуа кольца $R$, над которым многочлен $G(x)$ раскладывается (однозначно) на линейные множители. Пусть это разложение имеет вид $(7)$, и $G^{(d)}(x)-$ многочлен, определенный в (11).

Теорема 3. Пусть $u_{1}, \ldots, u_{K}$ - линейные рехурренты над хольцом $R$ с характеристическими многочленами $F_{1}(x), \ldots, F_{K}(x) \in R[x]$,

$$
\Phi\left(x_{1,1}, \ldots, x_{1, s_{1}}, \ldots, x_{K, 1}, \ldots, x_{K, s_{K}}\right)
$$

- многочлен над колъцом $R, s_{1}, \ldots, s_{K} \geqslant 0, r=\operatorname{deg} \Phi$,

$$
v(i)=\Phi\left(u_{1}(i), \ldots, u_{1}\left(i+s_{1}-1\right), \ldots, u_{K}(i), \ldots, u_{K}\left(i+s_{K}-1\right)\right), \quad i \geqslant 0,
$$

$D$ - множество чисел $d \geqslant 0$ таких, что в запись многочлена $\Phi$ входит с ненулевым коэфбициентом моном степени $d$.

Тогда многочлен

$$
H(x)=\prod_{d \in D} G^{(d)}(x)^{(a n-1) d+1},
$$

где $a=\max \left\{a\left(F_{1}\right), \ldots, a\left(F_{K}\right)\right\}, n=\operatorname{ind} J(R)$, является характеристическим многочленом ЛРП $v, u$

$$
\operatorname{rank} v \leqslant \sum_{d \in D}\left(\begin{array}{c}
m+d-1 \\
d
\end{array}\right)((a n-1) d+1) \leqslant\left(\begin{array}{c}
m+r \\
r
\end{array}\right)((a n-1) r+1)
$$

Если многочлены $F_{1}(x), \ldots, F_{K}(x)$ сепарабельны и попарно взаимно просты, то

$$
G(x)=F_{1}(x) \ldots F_{K}(x), \quad m=\operatorname{deg} F_{1}+\ldots+\operatorname{deg} F_{K},
$$

многочлен

$$
H_{1}(x)=\prod_{d \in D} G^{(d)}(x)
$$

является характеристическим многочленом ЛРП $v, u$

$$
\operatorname{rank} v \leqslant \sum_{d \in D}\left(\begin{array}{c}
m+d-1 \\
d
\end{array}\right) \leqslant\left(\begin{array}{c}
m+r \\
r
\end{array}\right)
$$

Доказательство. Последовательность $v$ есть линейная комбинация последовательностей

$$
w(i)=u_{k_{1}}\left(i+i_{1}\right) \ldots u_{k_{d}}\left(i+i_{d}\right), \quad i \geqslant 0,
$$

где $d \in D, 1 \leqslant k_{1}, \ldots, k_{d} \leqslant K, i_{1}, \ldots, i_{d} \geqslant 0$, с некоторыми коэффициентами, равными коэффициентам многочлена $\Phi$. Так как $\bar{F}_{k}(x) \mid \bar{G}_{k}(x)^{a}$ и $\bar{G}_{k}(x) \mid \bar{G}(x)$, по предложению $4 F_{k}(x) \mid G(x)^{a n}, k=1, \ldots, K$. Из доказательства предложения 5 следует, что ЛРП $u_{k}$ представляется в виде

$$
u_{k}=\sum_{r=1}^{m} \sum_{l=0}^{a n-1} c_{k r l} \vartheta_{r}^{[l]}, \quad c_{k r l} \in S, \quad k=1, \ldots, K
$$


Так же, как в предложении 6 , доказывается, что ЛРП $w$ аннулируется многочленом $G^{(d)}(x)^{(a n-1) d+1}$. Отсюда вытекает, что последовательность $v$ аннулируется многочленом $H(x)$.

Случай, когда многочлены $F_{1}(x), \ldots, F_{K}(x)$ сепарабельны и попарно взаимно просты, рассматривается аналогично.

Докажем еще одну, как правило более точную, верхнюю оценку ранга ЛРП $v$. Над кольцом $S$ многочлены $G_{k}(x)$ однозначно представляются в виде

$$
G_{k}(x)=\left(x-\vartheta_{k, 1}\right) \ldots\left(x-\vartheta_{k, m_{k}}\right), \quad m_{k}=\operatorname{deg} G_{k}(x), \quad k=1, \ldots, K .
$$

При $d_{1}, \ldots, d_{K} \geqslant 0$ введем обозначение

$$
G^{\left(d_{1}, \ldots, d_{K}\right)}(x)=\prod\left(x-\vartheta_{1, i_{11}} \ldots \vartheta_{1, i_{1 d_{1}}} \ldots \vartheta_{K, i_{K 1}} \ldots \vartheta_{K, i_{K d_{K}}}\right)
$$

где произведение берется по всем различным элементам

$$
\vartheta_{1, i_{11}} \ldots \vartheta_{1, i_{1 d_{1}}} \ldots \vartheta_{K, i_{K 1}} \ldots \vartheta_{K, i_{K d_{K}}}, \quad i_{k r} \geqslant 0, \quad r=1, \ldots, d_{k}, \quad k=1, \ldots, K
$$

кольца $S$. Многочлен $G^{\left(d_{1}, \ldots, d_{K}\right)}(x)$ можно также записать в виде

$$
G^{\left(d_{1}, \ldots, d_{K}\right)}(x)=\prod\left(x-\vartheta_{1,1}^{j_{1,1}} \ldots \vartheta_{1, m_{1}}^{j_{1, m_{1}}} \ldots \vartheta_{K, 1}^{j_{K, 1}} \ldots \vartheta_{K, m_{K}}^{j_{K, m_{K}}}\right)
$$

где произведение берется по всем различным элементам

$$
\begin{gathered}
\vartheta_{1,1}^{j_{1,1}} \ldots \vartheta_{1, m_{1}}^{j_{1, m_{1}}} \ldots \vartheta_{K, 1}^{j_{K, 1}} \ldots \vartheta_{K, m_{K}}^{j_{K, m_{K}}}, \\
j_{k, 1}+\ldots+j_{k, m_{k}}=d_{k}, \quad j_{k, r} \geqslant 0, \quad r=1, \ldots, m_{k}, \quad k=1, \ldots, K,
\end{gathered}
$$

кольца $S$. Аналогично (13) отсюда следует, что

$$
\operatorname{deg} G^{\left(d_{1}, \ldots, d_{K}\right)}(x) \leqslant\left(\begin{array}{c}
m_{1}+d_{1}-1 \\
d_{1}
\end{array}\right) \ldots\left(\begin{array}{c}
m_{K}+d_{K}-1 \\
d_{K}
\end{array}\right), \quad d_{1}, \ldots, d_{K} \geqslant 0 .
$$

Доказательство того, что $G^{\left(d_{1}, \ldots, d_{K}\right)}(x) \in R[x]$, основывается на следующем утверждении.

Предложение 7. Пусть $f\left(x_{1,1}, \ldots, x_{1, m_{1}}, \ldots, x_{K, 1}, \ldots, x_{K, m_{K}}\right)$ - многочлен над коммутативным колъцом $R$ с единичей, не меняющийся при произволъной перестановке переменных внутри каждого из векторов $\left(x_{k, 1}, \ldots, x_{k, m_{k}}\right), k=1, \ldots, K$.

Тогда $f$ полиномиалъно выражается через элементарные симметрические многочлена

$$
\sigma_{i}\left(x_{k, 1}, \ldots, x_{k, m_{k}}\right), \quad i=1, \ldots, m_{k}, \quad k=1, \ldots, K .
$$

Доказательство. Проведем индукцию по $K$. При $K=1$ утверждение совпадает с основной теоремой о симметрических многочленах. Проведем шаг индукции. Пусть

$$
f=f\left(\mathbf{x}_{1}, \mathbf{x}_{2}, \ldots, \mathbf{x}_{K}\right),
$$

где $\mathbf{x}_{k}=\left(x_{k, 1}, \ldots, x_{k, m_{k}}\right), k=1, \ldots, K$. Рассмотрим кольцо $S=R\left[\mathbf{x}_{2}, \ldots, \mathbf{x}_{K}\right]$. Тогда $f \in S\left[\mathbf{x}_{1}\right]$ - симметрический многочлен. По основной теореме о симметрических многочленах существует единственный многочлен $a\left(\mathbf{x}_{1}\right) \in S\left[\mathbf{x}_{1}\right]$ такой, что

$$
f\left(\mathbf{x}_{1}, \mathbf{x}_{2}, \ldots, \mathbf{x}_{K}\right)=a\left(\sigma_{1}\left(\mathbf{x}_{1}\right), \ldots, \sigma_{m_{1}}\left(\mathbf{x}_{1}\right)\right) .
$$


Записывая $a\left(\mathbf{x}_{1}\right)$ как многочлен $A\left(\mathbf{x}_{1}, \mathbf{x}_{2}, \ldots, \mathbf{x}_{K}\right)$ над кольцом $R$, получим, что

$$
f\left(\mathbf{x}_{1}, \mathbf{x}_{2}, \ldots, \mathbf{x}_{K}\right)=A\left(\sigma_{1}\left(\mathbf{x}_{1}\right), \ldots, \sigma_{m_{1}}\left(\mathbf{x}_{1}\right), \mathbf{x}_{2}, \ldots, \mathbf{x}_{K}\right) .
$$

Из последнего равенства следует, что многочлен $A\left(\sigma_{1}\left(\mathbf{x}_{1}\right), \ldots, \sigma_{m_{1}}\left(\mathbf{x}_{1}\right), \mathbf{x}_{2}, \ldots, \mathbf{x}_{K}\right)$ не меняется при перестановке переменных внутри каждого из векторов $\mathbf{x}_{2}, \ldots, \mathbf{x}_{K}$. Докажем, что и многочлен $A\left(\mathbf{x}_{1}, \mathbf{x}_{2}, \ldots, \mathbf{x}_{K}\right)$ обладает этим свойством. Действительно, в противном случае существуют перестановки $\mathbf{x}_{2}^{\prime}, \ldots, \mathbf{x}_{K}^{\prime}$ переменных такие, что

$$
A\left(\mathbf{x}_{1}, \mathbf{x}_{2}, \ldots, \mathbf{x}_{K}\right)
$$

и

$$
A^{\prime}\left(\mathbf{x}_{1}, \mathbf{x}_{2}, \ldots, \mathbf{x}_{K}\right)=A\left(\mathbf{x}_{1}, \mathbf{x}_{2}^{\prime}, \ldots, \mathbf{x}_{K}^{\prime}\right)
$$

- различные многочлены. Рассмотрим их как многочлены $a\left(\mathbf{x}_{1}\right)$ и $a^{\prime}\left(\mathbf{x}_{1}\right)$ над кольцом $S$. Тогда $a\left(\mathbf{x}_{1}\right) \neq a^{\prime}\left(\mathbf{x}_{1}\right)$, но

$$
a\left(\sigma_{1}\left(\mathbf{x}_{1}\right), \ldots, \sigma_{m_{1}}\left(\mathbf{x}_{1}\right)\right)=a^{\prime}\left(\sigma_{1}\left(\mathbf{x}_{1}\right), \ldots, \sigma_{m_{1}}\left(\mathbf{x}_{1}\right)\right),
$$

что противоречит утверждению о единственности в основной теореме о симметрических многочленах.

Рассмотрим кольцо $Q=R\left[\mathbf{x}_{1}\right]$. Тогда $A$ есть многочлен над кольцом $Q$ от переменных $\mathbf{x}_{2}, \ldots, \mathbf{x}_{K}$. По доказанному этот многочлен удовлетворяет предположению индукции. Поэтому существует многочлен $b\left(\mathbf{x}_{2}, \ldots, \mathbf{x}_{K}\right)$ над кольцом $Q$ такой, что

$$
A\left(\mathbf{x}_{1}, \mathbf{x}_{2}, \ldots, \mathbf{x}_{K}\right)=b\left(\sigma_{1}\left(\mathbf{x}_{2}\right), \ldots, \sigma_{m_{2}}\left(\mathbf{x}_{2}\right), \ldots, \sigma_{K}\left(\mathbf{x}_{K}\right), \ldots, \sigma_{m_{K}}\left(\mathbf{x}_{K}\right)\right) .
$$

Записывая $b\left(\mathbf{x}_{2}, \ldots, \mathbf{x}_{K}\right)$ как многочлен $B\left(\mathbf{x}_{1}, \mathbf{x}_{2}, \ldots, \mathbf{x}_{K}\right)$ над кольцом $R$, получим, что

$$
A\left(\mathbf{x}_{1}, \mathbf{x}_{2}, \ldots, \mathbf{x}_{K}\right)=B\left(\mathbf{x}_{1}, \sigma_{1}\left(\mathbf{x}_{2}\right), \ldots, \sigma_{m_{2}}\left(\mathbf{x}_{2}\right), \ldots, \sigma_{K}\left(\mathbf{x}_{K}\right), \ldots, \sigma_{m_{K}}\left(\mathbf{x}_{K}\right)\right) .
$$

Следовательно,

$$
\begin{aligned}
& f\left(\mathbf{x}_{1}, \mathbf{x}_{2}, \ldots, \mathbf{x}_{K}\right)=A\left(\sigma_{1}\left(\mathbf{x}_{1}\right), \ldots, \sigma_{m_{1}}\left(\mathbf{x}_{1}\right), \mathbf{x}_{2}, \ldots, \mathbf{x}_{K}\right) \\
& \quad=B\left(\sigma_{1}\left(\mathbf{x}_{1}\right), \ldots, \sigma_{m_{1}}\left(\mathbf{x}_{1}\right), \sigma_{1}\left(\mathbf{x}_{2}\right), \ldots, \sigma_{m_{2}}\left(\mathbf{x}_{2}\right), \ldots, \sigma_{K}\left(\mathbf{x}_{K}\right), \ldots, \sigma_{m_{K}}\left(\mathbf{x}_{K}\right)\right) .
\end{aligned}
$$

Теперь заметим, что для любого $k=1, \ldots, K$ многочлен $G^{\left(d_{1}, \ldots, d_{K}\right)}(x)$ не изменится при произвольной перестановке набора элементов $\vartheta_{k, 1}, \ldots, \vartheta_{k, m_{k}}$. В силу предложения 7 коэффициенты многочлена $G^{\left(d_{1}, \ldots, d_{K}\right)}(x)$ полиномиально выражаются через элементарные симметрические многочлены от $K$ указанных наборов элементов, то есть через коэффициенты многочленов $G_{1}(x), \ldots, G_{K}(x)$. Следовательно, $G^{\left(d_{1}, \ldots, d_{K}\right)}(x) \in R[x]$.

Предложение 8. Пусть $u_{k} \in L_{R}\left(F_{k}\right), a_{k}=a\left(F_{k}\right), d_{k} \geqslant 0, k=1, \ldots, K$. Тогда многочлен $G^{\left(d_{1}, \ldots, d_{K}\right)}(x)^{A}$, где

$$
A=\sum_{k=1}^{K}\left(\left(a_{k} n-1\right) d_{k}+1\right),
$$

является характеристическим многочленом последовательности

$$
w(i)=u_{1}\left(i+i_{1,1}\right) \ldots u_{1}\left(i+i_{1, d_{1}}\right) \ldots u_{K}\left(i+i_{K, 1}\right) \ldots u_{K}\left(i+i_{K, d_{K}}\right), \quad i \geqslant 0
$$


$\boldsymbol{u}$

$$
\operatorname{rank} w \leqslant\left(\begin{array}{c}
m_{1}+d_{1}-1 \\
d_{1}
\end{array}\right) \ldots\left(\begin{array}{c}
m_{K}+d_{K}-1 \\
d_{K}
\end{array}\right) \sum_{k=1}^{K}\left(\left(a_{k} n-1\right) d_{k}+1\right) .
$$

Если многочлены $F_{1}(x), \ldots, F_{K}(x)$ сепарабельны, по многочлен $G^{\left(d_{1}, \ldots, d_{K}\right)}(x)$ является характеристическим многочленом последовательности $w, u$

$$
\operatorname{rank} w \leqslant\left(\begin{array}{c}
m_{1}+d_{1}-1 \\
d_{1}
\end{array}\right) \ldots\left(\begin{array}{c}
m_{K}+d_{K}-1 \\
d_{K}
\end{array}\right) .
$$

Дохазательство. По предложению 5 ЛРП $u_{k}$ имеет биномиальное представление

$$
u_{k}=\sum_{r=1}^{m_{k}} \sum_{l=0}^{a_{k} n-1} c_{k r l} \vartheta_{k r}^{[l]}, \quad c_{k r l} \in S, \quad k=1, \ldots, K
$$

Тогда ЛРП $w$ есть линейная комбинация последовательностей вида

$$
\vartheta_{1, r_{11}}^{\left[l_{11}\right]}\left(i+i_{11}\right) \ldots \vartheta_{1, r_{1 d_{1}}}^{\left[l_{1 d_{1}}\right]}\left(i+i_{1 d_{1}}\right) \ldots \vartheta_{K, r_{K 1}}^{\left[l_{K 1}\right]}\left(i+i_{K 1}\right) \ldots \vartheta_{K, r_{K d_{K}}}^{\left[l_{K d_{K}}\right]}\left(i+i_{K d_{K}}\right) .
$$

Здесь $l_{k t} \leqslant a_{k} n-1$ при $1 \leqslant t \leqslant d_{k}, 1 \leqslant k \leqslant K$, поэтому $\sum_{k, t} l_{k t} \leqslant A-1$. По лемме 1 такая последовательность аннулируется многочленом

$$
\left(x-\vartheta_{1, r_{11}} \ldots \vartheta_{1, r_{1 d_{1}}} \ldots \vartheta_{K, r_{K 1}} \ldots \vartheta_{K, r_{K d_{K}}}\right)^{A}
$$

а значит, и многочленом $G^{\left(d_{1}, \ldots, d_{K}\right)}(x)^{A}$. Следовательно, ЛРП $w$ аннулируется многочленом $G^{\left(d_{1}, \ldots, d_{K}\right)}(x)^{A}$.

Если многочлены $F_{1}(x), \ldots, F_{K}(x)$ сепарабельны, то по предложению 5

$$
u_{k}=\sum_{r=1}^{m_{k}} c_{k r} \vartheta_{k r}^{[0]}, \quad c_{k r} \in S, \quad k=1, \ldots, K .
$$

Тогда ЛРП $w$ есть линейная комбинация последовательностей вида

$$
\vartheta_{1, r_{11}}^{[0]}\left(i+i_{11}\right) \ldots \vartheta_{1, r_{1 d_{1}}}^{[0]}\left(i+i_{1 d_{1}}\right) \ldots \vartheta_{K, r_{K 1}}^{[0]}\left(i+i_{K 1}\right) \ldots \vartheta_{K, r_{K d_{K}}}^{[0]}\left(i+i_{K d_{K}}\right)
$$

По лемме 1 такая последовательность аннулируется многочленом

$$
\left(x-\vartheta_{1, r_{11}} \ldots \vartheta_{1, r_{1 d_{1}}} \ldots \vartheta_{K, r_{K 1}} \ldots \vartheta_{K, r_{K d_{K}}}\right) \text {, }
$$

а значит, и многочленом $G^{\left(d_{1}, \ldots, d_{K}\right)}(x)$. Следовательно, ЛРП $w$ аннулируется многочленом $G^{\left(d_{1}, \ldots, d_{K}\right)}(x)$.

Указанные в формулировке оценки рангов вытекают из (26).

Теорема 4. Пусть $u_{1}, \ldots, u_{K}$ - линейнье рехурренты над кольчом $R$ с характеристическими многочленами $F_{1}(x), \ldots, F_{K}(x) \in R[x], a_{k}=a\left(F_{k}\right), n=\operatorname{ind} J(R)$,

$$
\Phi\left(x_{1,1}, \ldots, x_{1, s_{1}}, \ldots, x_{K, 1}, \ldots, x_{K, s_{K}}\right)
$$

- многочлен над кольчом $R, s_{1}, \ldots, s_{K} \geqslant 0$,

$$
v(i)=\Phi\left(u_{1}(i), \ldots, u_{1}\left(i+s_{1}-1\right), \ldots, u_{K}(i), \ldots, u_{K}\left(i+s_{K}-1\right)\right), \quad i \geqslant 0,
$$


$D$ - множество векторов $\left(d_{1}, \ldots, d_{K}\right) \in \mathbf{N}_{0}^{K}$ таких, что в запись многочлена $\Phi$ входит с ненулевим коэффичиентом моном, сумма степеней которого по переменным $x_{k, 1}, \ldots, x_{k, s_{k}}$ равна $d_{k}, k=1, \ldots, K$.

Тогда многочлен

$$
H(x)=\prod_{\left(d_{1}, \ldots, d_{K}\right) \in D} G^{\left(d_{1}, \ldots, d_{K}\right)}(x)^{\sum_{k=1}^{K}\left(\left(a_{k} n-1\right) d_{k}+1\right)}
$$

является характеристическим многочленом ЛРП $v, u$

$$
\operatorname{rank} v \leqslant \sum_{\left(d_{1}, \ldots, d_{K}\right) \in D}\left(\begin{array}{c}
m_{1}+d_{1}-1 \\
d_{1}
\end{array}\right) \ldots\left(\begin{array}{c}
m_{K}+d_{K}-1 \\
d_{K}
\end{array}\right) \sum_{k=1}^{K}\left(\left(a_{k} n-1\right) d_{k}+1\right) .
$$

Если многочлены $F_{1}(x), \ldots, F_{K}(x)$ сепарабельны, то многочлен

$$
H_{1}(x)=\prod_{\left(d_{1}, \ldots, d_{K}\right) \in D} G^{\left(d_{1}, \ldots, d_{K}\right)}(x)
$$

является характеристическим многочленом ЛРП $v, u$

$$
\operatorname{rank} v \leqslant \sum_{\left(d_{1}, \ldots, d_{K}\right) \in D}\left(\begin{array}{c}
m_{1}+d_{1}-1 \\
d_{1}
\end{array}\right) \ldots\left(\begin{array}{c}
m_{K}+d_{K}-1 \\
d_{K}
\end{array}\right)
$$

Теорема вытекает из предложения 8.

Отметим, что если многочлены $F_{1}(x), \ldots, F_{K}(x)$ сепарабельны и попарно взаимно просты, то оценка (30) сильнее оценки (20). Действительно, достаточно заметить, что в этом случае $m=m_{1}+\ldots+m_{K}$ и справедливо тождество

$$
\sum_{d_{1}+\ldots+d_{K}=d}\left(\begin{array}{c}
m_{1}+d_{1}-1 \\
d_{1}
\end{array}\right) \ldots\left(\begin{array}{c}
m_{K}+d_{K}-1 \\
d_{K}
\end{array}\right)=\left(\begin{array}{c}
m+d-1 \\
d
\end{array}\right) .
$$

Для его доказательства достаточно приравнять коэффициенты при $x^{d}$ в равенстве

$$
(1-x)^{-m_{1}} \ldots(1-x)^{-m_{K}}=(1-x)^{-m} .
$$

Кроме того, оценка (30) сильнее оценки (4), поскольку

$$
\left(\begin{array}{c}
m_{k}+d_{k}-1 \\
d_{k}
\end{array}\right) \leqslant m_{k}^{d_{k}}, \quad k=1, \ldots, K .
$$

Однако, в общем случае из оценок (4), (19), (29) любая может оказаться более сильной. Например, если

$$
F_{1}(x)=\ldots=F_{K}(x)=G(x)^{a}, \quad \Phi\left(x_{1}, \ldots, x_{k}\right)=x_{1} \ldots x_{k},
$$

To

$$
\operatorname{deg} G(x)=m=m_{1}=\ldots=m_{K}, \quad \operatorname{deg} F(x)=a m
$$

и правые части оценок (4), (19) и (29) имеют вид

$$
(a m)^{K}, \quad\left(\begin{array}{c}
m+K-1 \\
K
\end{array}\right)((a n-1) K+1), \quad m^{K}
$$

соответственно. Какая из оценок более точная, зависит от параметров $a, m, n, K$. 
Замечание 1. Результаты разделов 3 и 4 верны и для некоторых бесконечных колец. Так, все результаты сохраняются, если $R$ - локальное кольцо с нильпотентным радикалом и конечным полем вычетов. Примером такого кольца является $R=P\left[x_{1}, x_{2}, \ldots\right] / I$, где $P$ - конечное поле, $I$ - идеал, порожденный всеми мономами степени $n$.

Результаты разделов 3 и 4 сохраняются также в случае, когда $R$ - локальное кольцо с нильпотентным радикалом и совершенным полем вычетов. Единственное отличие заключается в том, что расширение Галуа $S$ кольца $R$, над которым многочлен $G(x)=\operatorname{rad} F(x)$ имеет разложение (7) на линейные множители, не обязательно имеет степень $\left[\operatorname{deg} G_{1}, \ldots, \operatorname{deg} G_{T}\right]$, как сказано выше в случае конечного поля вычетов $\bar{R}$.

Если $R$ - локальное кольцо с нильпотентным радикалом и произвольным полем вычетов, то результаты разделов 3 и 4 сохраняются при условии, что неприводимые над полем $\bar{R}$ многочлены $\bar{G}_{t}(x)$ и $\bar{G}_{k t}(x)$ в разложениях (5) и (18) сепарабельны, то есть не имеют кратных корней в поле разложения. В частности, полностью сохраняются результаты, сформулированные для случаев, когда многочлены $F(x)$ и $F_{1}(x), \ldots, F_{K}(x)$ сепарабельны. Для перечисленных выше классов колец формулировки и доказательства остаются без изменений. Отметим также, что ввиду свойств, перечисленных в конце разделе 2 , полученные результаты переносятся и на прямые суммы таких колец.

Если же $R$ - локальное кольцо с нильпотентным радикалом и не совершенным полем вычетов, и хотя бы один из многочленов $\bar{G}_{t}(x)$ (или $\bar{G}_{k t}(x)$ ) в разложении (5) (или (18)) не сепарабелен, то требуется по-другому определять уже понятие радикала $\operatorname{rad} \bar{F}(x)=\bar{G}(x)$ многочлена $\bar{F}(x)$ над полем $\bar{R}$ и изменять определение многочлена $G^{(d)}(x)$. Здесь мы не исследуем этот случай.

\section{5. Уточнение верхних оценок ранга}

Пусть $R$ - конечное локальное кольцо. В этом разделе при условии, что характеристический многочлен $F(x)$ ЛРП $u$ сепарабелен, мы приведем более точную оценку ранга ЛРП $v=\Phi(u)$. Аналогично при условии, что многочлены $F_{1}(x), \ldots, F_{K}(x)$ сепарабельны, $u_{k} \in L_{R}\left(F_{k}\right), k=1, \ldots, K$, мы укажем более точную оценку ранга ЛРП $v=\Phi\left(u_{1}, \ldots, u_{K}\right)$. В следующем разделе будут даны достаточные условия, при которых эти оценки достигаются.

Пусть $F(x) \in R[x]$ - унитарный сепарабельный многочлен степени $m \geqslant 1, S-$ расширение Галуа кольца $R$, над которым многочлен $F(x)$ (однозначно) раскладывается на линейные множители:

$$
F(x)=\left(x-\vartheta_{1}\right) \ldots\left(x-\vartheta_{m}\right), \quad \vartheta_{1}, \ldots, \vartheta_{m} \in S .
$$

Пусть $\bar{R}=\mathrm{GF}(q), q=p^{l}, p$ - простое число. Наша ближайшая цель - определить многочлены $F^{(d, \varepsilon)}(x)$, аналогичные многочленам $G^{(d)}(x)$ из $(11)$, но имеющие несколько меньшие степени (за счет чего получается более точная оценка ранга). Напомним, что если $F(x)$ сепарабелен, то, в обозначениях раздела $4, F(x)=G(x)$ и $G^{(d)}(x)=F^{(d)}(x)$. Предварительно введем ряд обозначений.

Пусть целое неотрицательное число $d$ имеет $p$-ичное разложение

$$
d=\sum_{r \geqslant 0} \nu_{r}(d) p^{r}, \quad 0 \leqslant \nu_{r}(d) \leqslant p-1, \quad r \geqslant 0 .
$$


Величину

$$
w_{p}(d)=\sum_{r \geqslant 0} \nu_{r}(d)
$$

назовем $p$-ичным весом числа $d$. Сумму $d=d_{1}+\ldots+d_{m}, d_{j} \geqslant 0$, будем называть $p$-разложением и записывать в виде

$$
d=d_{1}+\ldots+d_{m},
$$

если $\nu_{r}\left(d_{1}\right)+\ldots+\nu_{r}\left(d_{m}\right)=\nu_{r}(d)$ при всех $r \geqslant 0$, то есть, если при сложении чисел $d_{1}, \ldots, d_{m}$ в $p$-ичной системе счисления не возникает переносов. Это условие равносильно тому, что

$$
w_{p}\left(d_{1}\right)+\ldots+w_{p}\left(d_{m}\right)=w_{p}(d)
$$

(см. лемму 2(д) в [6]).

В общем случае, когда сумма $d=d_{1}+\ldots+d_{m}$ не обязательно является $p$ разложением,

$$
w_{p}\left(d_{1}\right)+\ldots+w_{p}\left(d_{m}\right)=w_{p}(d)+(p-1) \varepsilon
$$

для некоторого $\varepsilon \geqslant 0$ (см. лемму $2($ в, г) в [6]). Легко видеть, что $\varepsilon-$ число переносов, возникающих при сложении чисел $d_{1}, \ldots, d_{m}$ в $p$-ичной системе счисления.

При $d \geqslant 0, \varepsilon \geqslant 0$ определим многочлен

$$
F^{(d, \varepsilon)}(x)=\prod\left(x-\vartheta_{1}^{j_{1}} \ldots \vartheta_{m}^{j_{m}}\right)
$$

где произведение берется по всем различным элементам

$$
\vartheta_{1}^{j_{1}} \ldots \vartheta_{m}^{j_{m}}, \quad\left(j_{1}, \ldots, j_{m}\right) \in J_{p}(d, m, \varepsilon)
$$

кольца $S$, а множество $J_{p}(d, m, \varepsilon)$ определяется следующим образом:

$$
\begin{aligned}
J_{p}(d, m, \varepsilon)=\left\{\left(j_{1}, \ldots, j_{m}\right): j_{1}, \ldots, j_{m} \geqslant 0, j_{1}+\ldots+j_{m}=d,\right. \\
\\
\left.\left(w_{p}\left(j_{1}\right)+\ldots+w_{p}\left(j_{m}\right)-w_{p}(d)\right) /(p-1) \leqslant \varepsilon\right\} .
\end{aligned}
$$

Таким образом, множество $J_{p}(d, m, \varepsilon)$ состоит из всех векторов $\left(j_{1}, \ldots, j_{m}\right) \in \mathbf{N}_{0}^{m}$ таких, что $j_{1}+\ldots+j_{m}=d$ и при вычислении последней суммы в $p$-ичной системе счисления возникает не более $\varepsilon$ переносов. Положим

$$
F^{(d, \varepsilon)}(x)=e, \quad \varepsilon<0 .
$$

Так как элементы $\vartheta_{1}, \ldots, \vartheta_{m}$ входят в определение (32) симметрично, то так же, как для многочлена $G^{(d)}(x)$, показывается, что $F^{(d, \varepsilon)}(x) \in R[x]$.

Пусть $F^{(d)}(x)=G^{(d)}(x)$ - многочлен, определенный в (11). Следующие соотношения вытекают из определений многочленов $F^{(d, \varepsilon)}(x)$ и $F^{(d)}(x)$ :

$$
\begin{aligned}
& F^{(0, \varepsilon)}(x)=x-e, \quad F^{(1, \varepsilon)}(x)=F(x), \quad \varepsilon \geqslant 0, \\
& F^{(d, \varepsilon)}(x)=F^{(d)}(x), \quad 0 \leqslant d \leqslant p-1, \quad \varepsilon \geqslant 0, \\
& F^{(d, \varepsilon)}(x)\left|F^{(d, \varepsilon+1)}(x), \quad F^{(d, \varepsilon)}(x)\right| F^{(d)}(x), \quad d \geqslant 0, \quad \varepsilon \in \mathbf{Z} .
\end{aligned}
$$

Наряду с последним условием заметим, что $F^{(d, \varepsilon)}(x)=F^{(d)}(x)$ при достаточно больших $\varepsilon$. Действительно, из условия $j_{1}+\ldots+j_{m}=d$ следует, что

$$
\left(w_{p}\left(j_{1}\right)+\ldots+w_{p}\left(j_{m}\right)-w_{p}(d)\right) /(p-1) \leqslant\left(d-w_{p}(d)\right) /(p-1) .
$$


Поэтому при $\varepsilon \geqslant\left(d-w_{p}(d)\right) /(p-1)$ последнее условие в (33) лишнее, и согласно (32) и (12) $F^{(d, \varepsilon)}(x)=F^{(d)}(x)$.

Степень многочлена $F^{(d, \varepsilon)}(x)$ не превосходит мощности множества $J_{p}(d, m, \varepsilon)$. В частности, при $\varepsilon=0$ степень многочлена $F^{(d, 0)}(x)$ не превосходит числа наборов $\left(j_{1}, \ldots, j_{m}\right)$ таких, что $j_{1}+\ldots \dot{+} j_{m}=d$, то есть

$$
\nu_{r}\left(j_{1}\right)+\ldots+\nu_{r}\left(j_{m}\right)=\nu_{r}(d), \quad r \geqslant 0 .
$$

Отсюда получаем формулу

$$
\operatorname{deg} F^{(d, 0)}(x) \leqslant\left(\begin{array}{c}
m+\nu_{0}(d)-1 \\
\nu_{0}(d)
\end{array}\right)\left(\begin{array}{c}
m+\nu_{1}(d)-1 \\
\nu_{1}(d)
\end{array}\right) \ldots, \quad d \geqslant 0 .
$$

Отметим, что многочлен $\bar{F}^{(d, 0)}(x)$ над полем $\bar{R}=\operatorname{GF}(q)$ в случае, когда $\bar{F}(x)-$ многочлен максимального периода, рассматривался, например, в [19].

Для $a \in R$ пусть

$$
\begin{aligned}
& \lambda(a)=\max \left\{i \in \mathbf{N}_{0}: p^{i} a \neq 0\right\}, \quad a \neq 0, \\
& \lambda(0)=-1 .
\end{aligned}
$$

Отметим, что для любого $a \in R$ справедливо равенство

$$
\lambda(a)=\min \left\{i \in \mathbf{N}_{0}: p^{i} a=0\right\}-1 .
$$

Если $e-$ единица кольца $R$, то $p \bar{e}=\overline{0}$, следовательно, $p e \in J(R)$ и $p^{n} e=0$. Поэтому $\lambda(a)<n=\operatorname{ind} J(R)$. Более того, $\operatorname{char} R=p^{k}$ для некоторого $k=1, \ldots, n$, и тогда $\lambda(a)<k$ для любого $a \in R$.

Предложение 9. Пусть $u$ - ЛРП над конечным локальным колъцом $R$ с сепарабельным характеристическим многочленом $F(x)$ степени $m \geqslant 1, a \in R, d \geqslant 0$.

Тогда многочлен $F^{(d, \lambda(a))}(x)$ является характеристическим многочленом ЛРП $a u^{d}, u \operatorname{rank} a u^{d} \leqslant\left|J_{p}(d, m, \lambda(a))\right|$.

Доказательство. Если $a=0$, то $\lambda(a)=-1$ и $F^{(d,-1)}(x)=e$ - минимальный многочлен ЛРП $a u^{d}=0$. Если $a \neq 0, d=0$, то $\lambda(a) \geqslant 0$ и $F^{(0, \lambda(a))}(x)=x-e-$ минимальный многочлен ЛРП $a u^{d}=a$.

Пусть $a \neq 0, d>0$. В силу (31) и предложения 5 ЛРП $u$ имеет биномиальное представление (10). Тогда

$$
a u(i)^{d}=a\left(\sum_{r=1}^{m} c_{r} \vartheta_{r}^{i}\right)^{d}=a \sum_{j_{1}+\ldots+j_{m}=d} \frac{d !}{j_{1} ! \ldots j_{m} !} c_{1}^{j_{1}} \ldots c_{m}^{j_{m}}\left(\vartheta_{1}^{j_{1}} \ldots \vartheta_{m}^{j_{m}}\right)^{i} .
$$

В силу леммы 6.39 из [8] максимальная степень числа $p$, на которую делится полиномиальный коэффициент $d ! /\left(j_{1} ! \ldots j_{m} !\right)$, равна

$$
\left(w_{p}\left(j_{1}\right)+\ldots+w_{p}\left(j_{m}\right)-w_{p}(d)\right) /(p-1) .
$$

Поэтому при $\left(w_{p}\left(j_{1}\right)+\ldots+w_{p}\left(j_{m}\right)-w_{p}(d)\right) /(p-1)>\lambda(a)$ коэффициент в сумме (38) при биномиальной последовательности $\left(\vartheta_{1}^{j_{1}} \ldots \vartheta_{m}^{j_{m}}\right)^{i}$ равен 0 . Следовательно, с учетом (33), сумму (38) можно записать в виде

$$
a u(i)^{d}=a \sum_{\left(j_{1}, \ldots, j_{m}\right) \in J_{p}(d, m, \lambda(a))} \frac{d !}{j_{1} ! \ldots j_{m} !} c_{1}^{j_{1}} \ldots c_{m}^{j_{m}}\left(\vartheta_{1}^{j_{1}} \ldots \vartheta_{m}^{j_{m}}\right)^{i}, \quad i \geqslant 0 .
$$


В силу определения (32) каждая биномиальная последовательность в сумме (39) аннулируется многочленом $F^{(d, \lambda(a))}(x)$. Следовательно, и ЛРП $a u^{d}$ аннулируется многочленом $F^{(d, \lambda(a))}(x)$.

Теорема 5. Пусть и - ЛРП над конечным локальным кольцом $R$ с сепарабельным характеристическим многочленом $F(x)$ степени $m \geqslant 1$,

$$
\Phi(x)=\sum_{d \geqslant 0} \varphi_{d} x^{d} \in R[x] .
$$

Тогда многочлен

$$
F^{(\Phi)}(x)=\prod_{d \geqslant 0} F^{\left(d, \lambda\left(\varphi_{d}\right)\right)}(x)
$$

является характеристическим многочленом ЛРП $v=\Phi(u) u$

$$
\operatorname{rank} \Phi(u) \leqslant \sum_{d \geqslant 0}\left|J_{p}\left(d, m, \lambda\left(\varphi_{d}\right)\right)\right| .
$$

Доказательство. Так как

$$
v=\sum_{d \geqslant 0} \varphi_{d} u^{d}
$$

теорема следует из предложения 9 и определения многочлена $F^{(\Phi)}(x)$.

Для получения числовой оценки ранга ЛРП $\Phi(u)$ требуется найти $\left|J_{p}(d, m, \varepsilon)\right|$. Для этого введем понятие производного множества. Предшественником бесконечного целочисленного вектора

$$
\left(\nu_{0}, \nu_{1}, \ldots\right), \quad \nu_{j} \geqslant 0, \quad j \geqslant 0,
$$

лишь конечное число координат которого отлично от нуля, назовем произвольный вектор $\left(\nu_{0}^{\prime}, \nu_{1}^{\prime}, \ldots\right)$ такой, что для некоторого $i \geqslant 1$ выполняются соотношения

$$
\nu_{i} \geqslant 1, \quad \nu_{i}^{\prime}=\nu_{i}-1, \quad \nu_{i-1}^{\prime}=\nu_{i-1}+p, \quad \nu_{j}^{\prime}=\nu_{j}, \quad j \in \mathbf{N}_{0} \backslash\{i-1, i\} .
$$

Если $M$ - множество, состоящее из некоторых векторов вида (41), то множество, состоящее из всех предшественников векторов из $M$, обозначим $M^{\prime}$. В частности, если множество $M$ состоит из таких векторов (41), что $\nu_{j}=0$ при $j \geqslant 1$, то у этих векторов нет предшественников и $M^{\prime}=\varnothing$. Положим

$$
M^{(0)}=M, \quad M^{(e+1)}=\left(M^{(e)}\right)^{\prime}, \quad e \geqslant 1 .
$$

Легко видеть, что для любого $M$ существует $e \geqslant 0$ такое, что $M^{(e)}=\varnothing$.

При $d \geqslant 0$ введем обозначение

$$
M(d)=\left\{\left(\nu_{0}(d), \nu_{1}(d), \ldots\right)\right\} .
$$

Тогда множество $M(d)^{(e)}$ состоит из всех векторов вида (41) с $\nu_{0}+\nu_{1} p+\ldots=d$, для которых при вычислении последней суммы в $p$-ичной системе счисления возникает 
$e$ переносов. Это доказывается индукцией по $e \geqslant 0$ с использованием определения производного множества. Следовательно,

$$
\begin{aligned}
& J_{p}(d, m, \varepsilon)=\left\{\left(j_{1}, \ldots, j_{m}\right): j_{1}, \ldots, j_{m} \geqslant 0,\right. \\
& \left.\quad\left(\nu_{0}\left(j_{1}\right)+\ldots+\nu_{0}\left(j_{m}\right), \nu_{1}\left(j_{1}\right)+\ldots+\nu_{1}\left(j_{m}\right), \ldots\right) \in M(d)^{(e)}, 0 \leqslant e \leqslant \varepsilon\right\} .
\end{aligned}
$$

Для каждого целочисленного вектора $\left(\nu_{0}, \nu_{1}, \ldots\right)$ число векторов $\left(j_{1}, \ldots, j_{m}\right)$ таких, что $j_{1}, \ldots, j_{m} \geqslant 0$ и

$$
\begin{aligned}
& \nu_{0}\left(j_{1}\right)+\ldots+\nu_{0}\left(j_{m}\right)=\nu_{0}, \\
& \nu_{1}\left(j_{1}\right)+\ldots+\nu_{1}\left(j_{m}\right)=\nu_{1},
\end{aligned}
$$

равно

$$
\left\{\begin{array}{l}
m \\
\nu_{0}
\end{array}\right\}_{p}\left\{\begin{array}{l}
m \\
\nu_{1}
\end{array}\right\}_{p} \ldots
$$

где $\left\{\begin{array}{c}m \\ k\end{array}\right\}_{p}$ - число решений в целых числах уравнения

$$
y_{1}+\ldots+y_{m}=k, \quad 0 \leqslant y_{1}, \ldots, y_{m} \leqslant p-1,
$$

то есть число размещений $k$ одинаковых предметов по $m$ различным ячейкам при условии, что в каждую ячейку попадает не более $p-1$ предметов.

Множество $M(d)^{(e)}$ либо пусто (при достаточно больших $e$ ), либо состоит из векторов, вес (то есть сумма компонент) которых равен $w_{p}(d)+(p-1) e$. В частности, множества $M(d)^{(e)}, e \geqslant 0$, попарно не пересекаются. Теперь из (42) следует, что при $d \geqslant 0, \varepsilon \in \mathbf{Z}$

$$
\left|J_{p}(d, m, \varepsilon)\right|=\sum_{e=0}^{\varepsilon} \sum_{\left(\nu_{0}, \nu_{1}, \ldots\right) \in M(d)(e)}\left\{\begin{array}{l}
m \\
\nu_{0}
\end{array}\right\}_{p}\left\{\begin{array}{l}
m \\
\nu_{1}
\end{array}\right\}_{p} \ldots
$$

(при $\varepsilon<0$ сумма по пустому множеству индексов полагается равной 0 ). Так как в каждом векторе из $M(d)^{(e)}$ лишь конечное число координат отлично от нуля, произведение в (43) является конечным.

Согласно [10],

$$
\left\{\begin{array}{c}
m \\
k
\end{array}\right\}_{p}=\sum_{j \geqslant 0}(-1)^{j}\left(\begin{array}{c}
m \\
j
\end{array}\right)\left(\begin{array}{c}
m+k-p j-1 \\
m-1
\end{array}\right), \quad k \geqslant 0 .
$$

Справедливы неравенства

$$
\left(\begin{array}{c}
m \\
k
\end{array}\right) \leqslant\left\{\begin{array}{c}
m \\
k
\end{array}\right\}_{p} \leqslant\left(\begin{array}{c}
m+k-1 \\
k
\end{array}\right),
$$

поскольку в левой части находится число размещений $k$ одинаковых предметов по $m$ различным ячейкам при условии, что в каждую ячейку попадает не более одного предмета, а в правой части - число размещений $k$ предметов по $m$ ячейкам без ограничений на число предметов в ячейках. Заметим также, что

$$
\begin{array}{ll}
\left\{\begin{array}{l}
m \\
k
\end{array}\right\}_{p}=\left(\begin{array}{c}
m+k-1 \\
k
\end{array}\right), & k=0,1, \ldots, p-1, \\
\left\{\begin{array}{l}
m \\
k
\end{array}\right\}_{2}=\left(\begin{array}{c}
m \\
k
\end{array}\right), & p=2, \quad k \geqslant 0 .
\end{array}
$$


С учетом формулы (43) оценку (40) можно записать следующим образом.

Следствие 4. В условиях теоремъ 5

$$
\operatorname{rank} \Phi(u) \leqslant \sum_{d \geqslant 0} \sum_{e=0}^{\lambda\left(\varphi_{d}\right)} \sum_{\left(\nu_{0}, \nu_{1}, \ldots\right) \in M(d)^{(e)}}\left\{\begin{array}{l}
m \\
\nu_{0}
\end{array}\right\}_{p}\left\{\begin{array}{l}
m \\
\nu_{1}
\end{array}\right\}_{p} \ldots
$$

Отметим, что входящие в сумму (45) производные множества $M(d)^{(e)}$ легко строятся по множеству $M(d)=\left\{\left(\nu_{0}(d), \nu_{1}(d), \ldots\right)\right\}$.

Рассмотрим теперь случай нескольких последовательностей. Пусть

$$
F_{1}(x), \ldots, F_{K}(x)
$$

- сепарабельные многочлены над кольцом $R$ степеней $m_{1}, \ldots, m_{K}$ соответственно, $S$ - расширение Галуа кольца $R$, над которым многочлены $F_{1}(x), \ldots, F_{K}(x)$ (однозначно) раскладывается на линейные множители:

$$
F_{k}(x)=\left(x-\vartheta_{k, 1}\right) \ldots\left(x-\vartheta_{k, m_{k}}\right), \quad \vartheta_{k, 1}, \ldots, \vartheta_{k, m_{k}} \in S, \quad k=1, \ldots, K .
$$

При $d_{1}, \ldots, d_{K} \geqslant 0, \varepsilon \geqslant 0$ пусть

$$
F^{\left(d_{1}, \ldots, d_{K}, \varepsilon\right)}(x)=\prod\left(x-\vartheta_{1,1}^{j_{1,1}} \ldots \vartheta_{1, m_{1}}^{j_{1, m_{1}}} \ldots \vartheta_{K, 1}^{j_{K, 1}} \ldots \vartheta_{K, m_{K}}^{j_{K, m_{K}}}\right)
$$

где произведение берется по всем различным элементам

$$
\vartheta_{1,1}^{j_{11}} \ldots \vartheta_{1, m_{1}}^{j_{1 m_{1}}} \ldots \vartheta_{K, 1}^{j_{K 1}} \ldots \vartheta_{K, m_{K}}^{j_{K m_{K}}}
$$

кольца $S$ таким, что

$$
\left(j_{1,1}, \ldots, j_{1, m_{1}}, \ldots, j_{K, 1}, \ldots, j_{K, m_{K}}\right) \in J_{p}(\mathbf{d}, \mathbf{m}, \varepsilon)
$$

и

$$
\begin{aligned}
J_{p}(\mathbf{d}, \mathbf{m}, \varepsilon)= & J_{p}\left(d_{1}, \ldots, d_{K}, m_{1}, \ldots, m_{K}, \varepsilon\right) \\
= & \left(j_{1,1}, \ldots, j_{1, m_{1}}, \ldots, j_{K, 1}, \ldots, j_{K, m_{K}}\right): j_{k, r} \geqslant 0, \\
& j_{k, 1}+\ldots+j_{k, m_{k}}=d_{k}, \quad r=1, \ldots, m_{k}, \quad k=1, \ldots K, \\
& \left.\sum_{k=1}^{K}\left(w_{p}\left(j_{k, 1}\right)+\ldots+w_{p}\left(j_{k, m_{k}}\right)-w_{p}\left(d_{k}\right)\right) /(p-1) \leqslant \varepsilon\right\} .
\end{aligned}
$$

Таким образом, $J_{p}(\mathbf{d}, \mathbf{m}, \varepsilon)$ есть множество векторов $\left(j_{1,1}, \ldots, j_{K, m_{K}}\right)$ с целыми неотрицательными координатами такими, что $j_{k, 1}+\ldots+j_{k, m_{k}}=d_{k}$ при $k=1, \ldots, K$, и общее число переносов, возникающих при вычислении этих $K$ сумм в $p$-ичной системе счисления, не превосходит $\varepsilon$. Так как для каждого $k=1, \ldots, K$ элементы $\left\{\vartheta_{k, 1}, \ldots, \vartheta_{k, m_{k}}\right\}$ входят в определение (47) симметрично, то так же, как для многочленов $G^{\left(d_{1}, \ldots, d_{K}\right)}(x)$ в разделе 4 , доказывается, что $F^{\left(d_{1}, \ldots, d_{K}, \varepsilon\right)}(x) \in R[x]$. При $\varepsilon<0$ положим

$$
F^{\left(d_{1}, \ldots, d_{K}, \varepsilon\right)}(x)=e .
$$

Справедливы соотношения

$$
F^{\left(d_{1}, \ldots, d_{K}, \varepsilon\right)}(x)=F^{\left(d_{1}, \ldots, d_{K}\right)}(x), \quad 0 \leqslant d_{1}, \ldots, d_{K} \leqslant p-1, \quad \varepsilon \geqslant 0,
$$


И

$$
\begin{aligned}
& F^{\left(d_{1}, \ldots, d_{K}, \varepsilon\right)}(x) \mid F^{\left(d_{1}, \ldots, d_{K}, \varepsilon+1\right)}(x), \\
& F^{\left(d_{1}, \ldots, d_{K}, \varepsilon\right)}(x) \mid F^{\left(d_{1}, \ldots, d_{K}\right)}(x), \quad d_{1}, \ldots, d_{K} \geqslant 0, \quad \varepsilon \in \mathbf{Z} .
\end{aligned}
$$

При достаточно больших $\varepsilon$, зависящих от $p, d_{1}, \ldots, d_{K}$, выполняется равенство

$$
F^{\left(d_{1}, \ldots, d_{K}, \varepsilon\right)}(x)=F^{\left(d_{1}, \ldots, d_{K}\right)}(x) .
$$

Степень многочлена $F^{\left(d_{1}, \ldots, d_{K}, \varepsilon\right)}(x)$ при $\varepsilon \geqslant 0$ не превосходит мощности множества $J_{p}(\mathbf{d}, \mathbf{m}, \varepsilon)$. В частности, при $\varepsilon=0$ степень многочлена $F^{\left(d_{1}, \ldots, d_{K}, 0\right)}(x)$ не превосходит числа наборов

$$
\left(j_{1,1}, \ldots, j_{1, m_{1}}, \ldots, j_{K, 1}, \ldots, j_{K, m_{K}}\right)
$$

таких, что

$$
j_{k, 1}+\ldots+j_{k, m_{k}}=d_{k}, \quad k=1, \ldots, K,
$$

и, аналогично (37), удовлетворяет неравенству

$$
\operatorname{deg} F^{\left(d_{1}, \ldots, d_{K}, 0\right)}(x) \leqslant \prod_{k=1}^{K}\left(\begin{array}{c}
m_{k}+\nu_{0}\left(d_{k}\right)-1 \\
\nu_{0}\left(d_{k}\right)
\end{array}\right)\left(\begin{array}{c}
m_{k}+\nu_{1}\left(d_{k}\right)-1 \\
\nu_{1}\left(d_{k}\right)
\end{array}\right) \ldots
$$

Предложение 10. Пусть $u_{1}, \ldots, u_{K}$ - линейные рекурренты над конечным локальным хольчом $R$ с сепарабельнъми харахтеристическими многочленами $F_{1}(x), \ldots, F_{K}(x) \in R[x]$ степеней $m_{1}, \ldots, m_{K} \geqslant 1$ соответственно, $a \in R, d_{1}, \ldots, d_{K}$ неотрицательны.

Тогда многочлен $F^{\left(d_{1}, \ldots, d_{k}, \lambda(a)\right)}(x)$ является характеристическим многочленом ЛРП $w=a u_{1}^{d_{1}} \ldots u_{K}^{d_{K}} u \operatorname{rank} w \leqslant\left|J_{p}(\mathbf{d}, \mathbf{m}, \lambda(a))\right|$.

Доказательство. В силу (46) и предложения 5 последовательности $u_{1}, \ldots, u_{K}$ представляются в виде

$$
u_{k}(i)=\sum_{r=1}^{m_{k}} c_{k r} \vartheta_{k r}^{i}, \quad i \geqslant 0, \quad c_{k r} \in S, \quad k=1, \ldots, K .
$$

Тогда

$$
\begin{aligned}
& w(i)=a\left(\sum_{r=1}^{m_{1}} c_{1, r} \vartheta_{1, r}^{i}\right)^{d_{1}} \ldots\left(\sum_{r=1}^{m_{K}} c_{K, r} \vartheta_{K, r}^{i}\right)^{d_{K}} \\
& =a \sum_{j_{k, 1}+\ldots+j_{k, m_{k}}=d_{k}} \frac{d_{1} !}{j_{1,1} ! \ldots j_{1, m_{1}} !} \cdots \frac{d_{K} !}{j_{K, 1} ! \ldots j_{K, m_{K}} !} \\
& \times c_{1,1}^{j_{1,1}} \ldots c_{1, m_{1}}^{j_{1, m_{1}}} \ldots c_{K, 1}^{j_{K, 1}} \ldots c_{K, m_{K}}^{j_{K, m_{K}}}\left(\vartheta_{1,1}^{j_{1,1}} \ldots \vartheta_{1, m_{1}}^{j_{1, m_{1}}} \ldots \vartheta_{K, 1}^{j_{K, 1}} \ldots \vartheta_{K, m_{K}}^{j_{K, m_{K}}}\right)^{i},
\end{aligned}
$$

где суммирование в последней сумме проводится по

$$
j_{k, 1}, \ldots, j_{k, m_{k}} \geqslant 0, \quad 1 \leqslant k \leqslant K .
$$

В силу леммы 6.39 из [8] максимальная степень числа $p$, на которую делится произведение полиномиальных коэффициентов в сумме (53), равна

$$
E=\sum_{k=1}^{K}\left(w_{p}\left(j_{k, 1}\right)+\ldots+w_{p}\left(j_{k, m_{k}}\right)-w_{p}\left(d_{k}\right)\right) /(p-1) .
$$


Поэтому при $E>\lambda(a)$ соответствующее слагаемое в сумме (53) равно 0. Следовательно, с учетом (48),

$$
\begin{aligned}
w(i)=a & \sum_{\left(j_{1,1}, \ldots, j_{K, m_{K}}\right) \in J_{p}(\mathbf{d}, \mathbf{m}, \lambda(a))} \frac{d_{1} !}{j_{1,1} ! \ldots j_{1, m_{1}} !} \ldots \frac{d_{K} !}{j_{K, 1} ! \ldots j_{K, m_{K}} !} \\
& \times c_{1,1}^{j_{1,1}} \ldots c_{1, m_{1}}^{j_{1, m_{1}}} \ldots c_{K, 1}^{j_{K, 1}} \ldots c_{K, m_{K}}^{j_{K, m_{K}}}\left(\vartheta_{1,1}^{j_{1,1}} \ldots \vartheta_{1, m_{1}}^{j_{1, m_{1}}} \ldots \vartheta_{K, 1}^{j_{K, 1}} \ldots \vartheta_{K, m_{K}}^{\left.j_{K, m_{K}}\right)^{i}} .\right.
\end{aligned}
$$

В силу определения (47) каждая биномиальная последовательность в сумме (54) аннулируется многочленом $F^{\left(d_{1}, \ldots, d_{k}, \lambda(a)\right)}(x)$. Следовательно, и последовательность $w$ аннулируется этим многочленом.

Для многочлена

$$
\Phi\left(x_{1}, \ldots, x_{K}\right)=\sum_{d_{1}, \ldots, d_{K} \geqslant 0} \varphi_{d_{1} \ldots d_{K}} x_{1}^{d_{1}} \ldots x_{K}^{d_{K}}
$$

над кольцом $R$ положим

$$
F^{(\Phi)}(x)=\prod_{d_{1}, \ldots, d_{K} \geqslant 0} F^{\left(d_{1}, \ldots, d_{K}, \lambda\left(\varphi_{d_{1} \ldots d_{K}}\right)\right)}(x) .
$$

Теорема 6. Пусть $u_{1}, \ldots, u_{K}$ - линейные рекурренты над конечным локальным кольцом $R$ c сепарабелъными характеристическими многочленами $F_{1}(x), \ldots, F_{K}(x) \in R[x]$ степеней $m_{1}, \ldots, m_{K} \geqslant 1$ соответственно,

$$
\Phi\left(x_{1}, \ldots, x_{K}\right) \in R\left[x_{1}, \ldots, x_{K}\right] .
$$

Тогда многочлен $F^{(\Phi)}(x)$ является характеристическим многочленом ЛРП $v=$ $\Phi\left(u_{1}, \ldots, u_{K}\right), u$

$$
\operatorname{rank} \Phi\left(u_{1}, \ldots, u_{K}\right) \leqslant \sum_{d_{1}, \ldots, d_{K} \geqslant 0}\left|J_{p}\left(\mathbf{d}, \mathbf{m}, \lambda\left(\varphi_{d_{1} \ldots d_{K}}\right)\right)\right| .
$$

Теорема следует из предложения 10.

Получим числовую оценку ранга ЛРП $\Phi\left(u_{1}, \ldots, u_{K}\right)$. В силу $(33),(48)$

$$
\begin{aligned}
& J_{p}(\mathbf{d}, \mathbf{m}, \varepsilon)=\left\{\left(j_{1,1}, \ldots, j_{1, m_{1}}, \ldots, j_{K, 1}, \ldots, j_{K, m_{K}}\right):\right. \\
& \left.\quad\left(j_{k, 1}, \ldots, j_{k, m_{k}}\right) \in J_{p}\left(d_{k}, m_{k}, e_{k}\right), e_{k} \geqslant 0, k=1, \ldots, K, e_{1}+\ldots+e_{K} \leqslant \varepsilon\right\} .
\end{aligned}
$$

Используя (42), получим, что

$$
\begin{aligned}
J_{p}(\mathbf{d}, \mathbf{m}, \varepsilon)=\{ & \left(j_{1,1}, \ldots, j_{1, m_{1}}, \ldots, j_{K, 1}, \ldots, j_{K, m_{K}}\right): \\
& \left(\nu_{0}\left(j_{k, 1}\right)+\ldots+\nu_{0}\left(j_{k, m_{k}}\right), \nu_{1}\left(j_{k, 1}\right)+\ldots+\nu_{1}\left(j_{k, m_{k}}\right), \ldots\right) \in M\left(d_{k}\right)^{\left(e_{k}\right)}, \\
& \left.j_{k, r} \geqslant 0, \quad e_{k} \geqslant 0, \quad r=1, \ldots, m_{k}, \quad k=1, \ldots, K, \quad e_{1}+\ldots+e_{K} \leqslant \varepsilon\right\} \\
= & \bigcup_{\substack{e_{1}, \ldots, e_{K} \geqslant 0 \\
e_{1}+\ldots+e_{K} \leqslant \varepsilon}}\left\{\left(j_{1,1}, \ldots, j_{1, m_{1}}, \ldots, j_{K, 1}, \ldots, j_{K, m_{K}}\right): j_{k, r} \geqslant 0, r=1, \ldots, m_{k},\right. \\
& \left(\nu_{0}\left(j_{k, 1}\right)+\ldots+\nu_{0}\left(j_{k, m_{k}}\right), \nu_{1}\left(j_{k, 1}\right)+\ldots+\nu_{1}\left(j_{k, m_{k}}\right), \ldots\right) \in M\left(d_{k}\right)^{\left(e_{k}\right)}, \\
& k=1, \ldots, K\},
\end{aligned}
$$


причем в последнем соотношении объединяемые множества попарно не пересекаются, поскольку множества $M(d)^{(e)}, e \geqslant 0$, попарно не пересекаются. Следовательно, при $d_{1}, \ldots, d_{K} \geqslant 0, \varepsilon \geqslant 0$ аналогично (43) получаем равенство

$$
\left|J_{p}(\mathbf{d}, \mathbf{m}, \varepsilon)\right|=\sum_{\substack{e_{1}, \ldots, e_{K} \geqslant 0 \\
e_{1}+\ldots+e_{K} \leqslant \varepsilon}} \sum_{\substack{\left(\nu_{k 0}, \nu_{k 1}, \ldots, \in M\left(d_{k}\right)^{\left(e_{k}\right)} \\
k=1, \ldots, K\right.}} \prod_{k=1}^{K}\left\{\begin{array}{l}
m_{k} \\
\nu_{k 0}
\end{array}\right\}_{p}\left\{\begin{array}{l}
m_{k} \\
\nu_{k 1}
\end{array}\right\}_{p} \ldots
$$

Поскольку в каждом векторе из $M\left(d_{k}\right)^{\left(e_{k}\right)}$ лишь конечное число координат отлично от нуля, последнее произведение является конечным.

Следствие 5. В условиях теореми 6

$$
\begin{aligned}
& \operatorname{rank} \Phi\left(u_{1}, \ldots, u_{K}\right) \\
& \leqslant \sum_{d_{1}, \ldots, d_{K} \geqslant 0} \sum_{\substack{e_{1}+\ldots+e_{K} \leqslant \lambda\left(\varphi_{\left.d_{1} \ldots d_{K}\right)} \\
e_{1}, \ldots, e_{K} \geqslant 0\right.}} \sum_{\substack{\left(\nu_{k 0}, \nu_{k 1}, \ldots\right) \in M\left(d_{k}\right) \\
k=1, \ldots, K}} \prod_{\substack{\left(e_{k}\right) \\
k=1}}^{K}\left\{\begin{array}{l}
m_{k} \\
\nu_{k 0}
\end{array}\right\}_{p}\left\{\begin{array}{l}
m_{k} \\
\nu_{k 1}
\end{array}\right\}_{p} \ldots
\end{aligned}
$$

Напомним, что величины $\left\{\begin{array}{c}m \\ k\end{array}\right\}_{p}$ в этой оценке вычисляются по формуле (44).

Замечание 2. Формулировки и доказательства результатов этого параграфа сохраняются и в случае, когда $R$ - локальное кольцо с нильпотентным радикалом, поле вычетов которого имеет характеристику $p>0$.

\section{6. Минимальный многочлен и точное значение ранга}

В этом параграфе будет доказано, что при дополнительных условиях на последовательности $u, u_{1}, \ldots, u_{K}$ и многочлен $\Phi$ многочлены $F^{(\Phi)}(x)$, определенные в теоремах 5 и 6 , являются минимальными многочленами последовательностей $\Phi(u)$ и $\Phi\left(u_{1}, \ldots, u_{K}\right)$, и верхние оценки рангов, полученные в следствиях 4 и 5 , обращаются в равенства.

Пусть $R$ - конечное локальное кольцо с полем вычетов $\bar{R}=\mathrm{GF}(q), F(x) \in R[x]$ - многочлен Галуа степени $m, S$ - расширение Галуа кольца $R$ степени $m$. Над кольцом $S$ многочлен $F(x)$ представляется в виде (31), причем, если обозначить $\vartheta=\vartheta_{1}$, то элементы $\vartheta_{2}, \ldots, \vartheta_{m}$ можно занумеровать так, что

$$
\bar{\vartheta}_{r}=\bar{\vartheta}^{r-1}, \quad r=1, \ldots, m
$$

Напомним, что неприводимый многочлен $\bar{F}(x)$ над полем $\bar{R}$ называется многочленом максимального периода или примитивным многочленом, если его корень $\bar{\vartheta}$ является примитивным элементом поля $\bar{S}=\mathrm{GF}\left(q^{m}\right)$, то есть ord $\bar{\vartheta}=q^{m}-1$.

Предложение 11. Пусть $F(x) \in R[x]$ - многочлен Галуа степени $m \geqslant 1$ тахой, что $\bar{F}(x)$ - многочлен махсимального периода над полем $\bar{R}$. Тогда выполняются следующие утверждения. 
При $d \doteq 0,1, \ldots, q-1$ справедливъ равенства

$$
F^{(d)}(x)=\prod_{\substack{j_{1}+\ldots+j_{m}=d \\
j_{1}, \ldots, j_{m} \geqslant 0}}\left(x-\vartheta_{1}^{j_{1}} \ldots \vartheta_{m}^{j_{m}}\right), \quad \operatorname{deg} F^{(d)}(x)=\left(\begin{array}{c}
m+d-1 \\
d
\end{array}\right)
$$

Многочлены $F^{(1)}(x), \ldots, F^{(q-1)}(x)$ сепарабелъны и попарно взаимно просты.

Если $m \geqslant 2$, то многочлены $F^{(0)}(x)=x-e, F^{(1)}(x), \ldots, F^{(q-1)}(x)$ попарно взаимно просты.

Доказательство. Так как $\bar{F}(x)$ - многочлен максимального периода, элементы

$$
\bar{\vartheta}_{1}^{j_{1}} \ldots \bar{\vartheta}_{m}^{j_{m}}=\bar{\vartheta}^{j_{1}+q j_{2}+\ldots+q^{m-1} j_{m}}
$$

при $0 \leqslant j_{1}, \ldots, j_{m} \leqslant q-1,\left(j_{1}, \ldots, j_{m}\right) \neq(0, \ldots, 0)$ попарно различны, при этом

$$
\bar{\vartheta}_{1}^{0} \ldots \bar{\vartheta}_{m}^{0}=\bar{e}=\bar{\vartheta}_{1}^{q-1} \ldots \bar{\vartheta}_{m}^{q-1}
$$

Теперь первое утверждение вытекает из определения (12). Второе и третье утверждения вытекают из того, что элементы (59) попарно различны.

Следующее утверждение доказано в [9].

Предложение 12. Пусть ЛРП $u_{1}, \ldots, u_{s}$ над колъцом $R$ имеют попарно взаимно простые харахтеристические многочлены, $u=u_{1}+\ldots+u_{s}$. Тогда произведение минимальных многочленов ЛРП $u_{1}, \ldots, u_{s}$ является минимальным многочленом ЛРП $u u \operatorname{rank} u=\operatorname{rank} u_{1}+\ldots+\operatorname{rank} u_{s}$.

Предложение 13. Пусть $F(x) \in R[x]$ - многочлен Галуа степени $m \geqslant 1$ такой, что $\bar{F}(x)$ - многочлен максимального периода над полем $\bar{R}, u \in L_{R}(F), \bar{u} \neq 0, a \in$ $R, 0 \leqslant d \leqslant q-1$. Тогда многочлен $F^{(d, \lambda(a))}(x)$ лвляется минималъным многочленом ЛРП $a u^{d} u \operatorname{rank} a u^{d}=\left|J_{p}(d, m, \lambda(a))\right|$.

Доказательство. Поскольку выполняются условия предложения 9, можно использовать соотношение (39). Как следует из доказательства предложения 9, для каждого слагаемого суммы (39) выполняется условие $a d ! /\left(j_{1} ! \ldots j_{m} !\right) \neq 0$. Так как $\bar{u} \neq 0$, в биномиальном представлении (10) $\bar{c}_{r} \neq 0, r=1, \ldots, m$, откуда, $\bar{c}_{1}^{j_{1}} \ldots \bar{c}_{m}^{j_{m}} \neq 0$. Следовательно, в сумме (39) коэффициент при каждой биномиальной последовательности $\left(\vartheta_{1}^{j_{1}} \ldots \vartheta_{m}^{j_{m}}\right)^{i}$ не равен 0 .

Таким образом, соотношение (39) есть представление ЛРП $a u^{d}$ в виде суммы последовательностей с минимальными многочленами

$$
x-\vartheta_{1}^{j_{1}} \ldots \vartheta_{m}^{j_{m}}, \quad\left(j_{1}, \ldots, j_{m}\right) \in J_{p}(d, m, \lambda(a))
$$

Из доказательства предложения 11 и условия $d \leqslant q-1$ следует, что элементы $\bar{\vartheta}_{1}^{j_{1}} \ldots \bar{\vartheta}_{m}^{j_{m}}$ поля $\bar{S}$ такие, что $\left(j_{1}, \ldots, j_{m}\right) \in J_{p}(d, m, \lambda(a))$, попарно различны. Следовательно, ввиду второго утверждения предложения 3, указанные минимальные многочлены попарно взаимно просты. Теперь из (39) и предложения 12 получаем, что $F^{(d, \lambda(a))}(x)$ - минимальный многочлен $\operatorname{JPП~} a u^{d}$ и $\operatorname{rank} a u^{d}=\left|J_{p}(d, m, \lambda(a))\right|$. 
Теорема 7. Пусть $R$ - конечное локальное кольчо, $F(x) \in R[x]-$ многочлен Галуа степени $m \geqslant 1$ тахой, что $\bar{F}(x)$ - многочлен махсимального периода над полем $\bar{R}, u \in L_{R}(F), \bar{u} \neq 0, \Phi(x) \in R[x]$ - многочлен степени не выше $q-1$ и либо $m \geqslant 2$, либо $m=1, \Phi(0)=0$.

Тогда многочлен

$$
F^{(\Phi)}(x)=\prod_{d \geqslant 0} F^{\left(d, \lambda\left(\varphi_{d}\right)\right)}(x)
$$

из теоремы 5 является минимальным многочленом ЛРП $v=\Phi(u)$ и оченхи (40), (45) обращаются в равенства.

Доказательство. Пусть

$$
\Phi(x)=\sum_{d=0}^{q-1} \varphi_{d} x^{d}
$$

Тогда

$$
v=\sum_{d=0}^{q-1} \varphi_{d} u^{d}
$$

По предложению 13 многочлен $F^{\left(d, \lambda\left(\varphi_{d}\right)\right)}(x)$ является минимальным многочленом ЛРП $\varphi_{d} u^{d}, d=0,1, \ldots, q-1$. Ввиду $(36) F^{\left(d, \lambda\left(\varphi_{d}\right)\right)}(x) \mid F^{(d)}(x)$. Если $m \geqslant 2$, то из третьего утверждения в 11 следует, что многочлены $F^{\left(d, \lambda\left(\varphi_{d}\right)\right)}(x), d=0,1, \ldots, q-1$, попарно взаимно просты. Если же $m=1$, то по условию $\varphi_{0}=0$, откуда, $\lambda\left(\varphi_{0}\right)=-1$ и $F^{\left(0, \lambda\left(\varphi_{0}\right)\right)}(x)=e$. В этом случае, в силу второго утверждения предложения 11 , многочлены $F^{\left(d, \lambda\left(\varphi_{d}\right)\right)}(x), d=0,1, \ldots, q-1$, также попарно взаимно просты. Из предложения 12 теперь следует, что произведение минимальных многочленов последовательностей $\varphi_{d} u^{d}, d=0,1, \ldots, q-1$, то есть многочлен $F^{(\Phi)}(x)$, является минимальным многочленом ЛРП $v$.

Вычисление ранга ЛРП $v=\Phi(u)$ с помощью формулы (45) сопряжено с построением производных множеств $M(d)^{(e)}, e \geqslant 0$. Укажем оценки ранга ЛРП $v$, которые являются более простыми и не требуют построения производных множеств.

Следствие 6. В условиях теоремъ 7 пусть $D=\left\{d \geqslant 0: \varphi_{d} \neq 0\right\}$. Тогда

$$
\sum_{d \in D}\left(\begin{array}{c}
m+\nu_{0}(d)-1 \\
\nu_{0}(d)
\end{array}\right)\left(\begin{array}{c}
m+\nu_{1}(d)-1 \\
\nu_{1}(d)
\end{array}\right) \ldots \leqslant \operatorname{rank} v \leqslant \sum_{d \in D}\left(\begin{array}{c}
m+d-1 \\
d
\end{array}\right) .
$$

Доказательство. Если $d \in D$, то $\lambda\left(\varphi_{d}\right) \geqslant 0$ и ввиду $(36) F^{(d, 0)}(x) \mid F^{\left(d, \lambda\left(\varphi_{d}\right)\right)}(x)$. Следовательно,

$$
\prod_{d \in D} F^{(d, 0)}(x) \mid F^{(\Phi)}(x)
$$

Поэтому

$$
\operatorname{rank} v=\operatorname{deg} F^{(\Phi)}(x) \geqslant \sum_{d \in D} \operatorname{deg} F^{(d, 0)}(x) .
$$

Так как элементы (59) попарно различны, неравенство (37) обращается в равенство. Отсюда получаем нижнюю оценку в (60). Верхняя оценка совпадает с оценкой (16), доказанной в теореме 2. 
Следствие 7. Если в условиях теоремь $7 \operatorname{deg} \Phi \leqslant p-1$, mо

$$
F^{(\Phi)}(x)=\prod_{d \in D} F^{(d)}(x), \quad \operatorname{rank} v=\sum_{d \in D}\left(\begin{array}{c}
m+d-1 \\
d
\end{array}\right)
$$

то есть для ранга достигается оченка (16).

Утверждения следствия вытекают из формул (35), (58).

Следствие 8. Пусть в условиях теоремы $7 R-$ колъцо главных идеалов с радикалом $J(R)=\pi R$ индекса нильпотентности п. Тогда

$$
\operatorname{Ann}(v)=\left(F^{(\Phi)}(x), \pi F^{(\pi \Phi)}(x), \ldots, \pi^{n-1} F^{\left(\pi^{n-1} \Phi\right)}(x)\right) .
$$

Доказателъство. По теореме 7 многочлен $F^{\left(\pi^{t} \Phi\right)}(x)$ является минимальным многочленом последовательности $\pi^{t} v, t=0,1, \ldots, n-1$. Другими словами, $F^{\left(\pi^{t} \Phi\right)}(x)-$ многочлен наименьшей степени среди унитарных многочленов $G(x) \in R[x]$ таких, что $\pi^{t} G(x) \in \operatorname{Ann}(v)$. Из утверждения 13 в [9] следует, что идеал $\operatorname{Ann}(v)$ порождается многочленами

$$
\pi^{t} F^{\left(\pi^{t} \Phi\right)}(x), \quad t=0,1, \ldots, n-1,
$$

что и требовалось доказать.

Теорема 7 и ее следствия справедливы, в частности, если $R=\operatorname{GR}\left(q^{n}, p^{n}\right)-$ кольцо Галуа из $q^{n}$ элементов характеристики $p^{n}$. Отметим, что в этом случае

$$
\lambda(a)=n-\|a\|-1,
$$

где

$$
\|a\|=\max \left\{r \in\{0, \ldots, n\}: a \in p^{r} R\right\}
$$

- норма элемента $a \in R$ (см. $[19,9])$.

Рассмотрим теперь полиномиальные преобразования нескольких последовательностей. Пусть $F_{1}(x), \ldots, F_{K}(x)$ - многочлены Галуа над $R$ степеней $m_{1}, \ldots, m_{K}$ соответственно. Над расширением Галуа $S$ кольца $R$ степени $\left[m_{1}, \ldots, m_{K}\right]$ многочлены $F_{1}(x), \ldots, F_{K}(x)$ представляются в виде $(46)$, причем если положить $\vartheta_{k}=\vartheta_{k, 1}$, то корни многочлена $F_{k}(x)$ можно занумеровать так, что

$$
\bar{\vartheta}_{k, r}=\bar{\vartheta}_{k}^{q^{r-1}}, \quad r=1, \ldots, m_{k}, \quad k=1, \ldots, K
$$

Положим

$$
M_{k}=\min \left\{q, m_{k}, \frac{q-2}{q-1} m_{k}+1\right\}, \quad k=1, \ldots, K .
$$

Предложение 14. Пусть $F_{1}(x), \ldots, F_{K}(x)$ - многочленъ Галуа над кольцом $R$ степеней $m_{1}, \ldots, m_{K} \geqslant 1$ соответственно такие, что $\bar{F}_{1}(x), \ldots, \bar{F}_{K}(x)$ - многочленъ максималъного периода над полем $\bar{R}$, числа $m_{1}, \ldots, m_{K}$ попарно взаимно просты $u 0 \leqslant d_{k}<M_{k}, k=1, \ldots, K$.

Tогда

$$
F^{\left(d_{1}, \ldots, d_{K}\right)}(x)=\prod\left(x-\vartheta_{1,1}^{j_{1,1}} \ldots \vartheta_{1, m_{1}}^{j_{1, m_{1}}} \ldots \vartheta_{K, 1}^{j_{K, 1}} \ldots \vartheta_{K, m_{K}}^{j_{K, m_{K}}}\right)
$$

где произведение берется по множеству

$$
\left\{j_{k, 1}, \ldots, j_{k, m_{k}}: j_{k, 1}+\ldots+j_{k, m_{k}}=d_{k}, j_{k, r} \geqslant 0,1 \leqslant r \leqslant m_{k}, 1 \leqslant k \leqslant K\right\}
$$


$\boldsymbol{u}$

$$
\operatorname{deg} F^{\left(d_{1}, \ldots, d_{K}\right)}(x)=\left(\begin{array}{c}
m_{1}+d_{1}-1 \\
d_{1}
\end{array}\right) \ldots\left(\begin{array}{c}
m_{K}+d_{K}-1 \\
d_{K}
\end{array}\right)
$$

многочлень

$$
F^{\left(d_{1}, \ldots, d_{K}\right)}(x), \quad 0 \leqslant d_{k}<M_{k}, \quad k=1, \ldots, K,
$$

сепарабельны и попарно взаимно просты.

Доказателъство. Ввиду определения (24) перечисленные утверждения являются следствием того, что элементы

$$
\bar{\vartheta}_{1,1}^{j_{1,1}} \ldots \bar{\vartheta}_{1, m_{1}}^{j_{1, m_{1}}} \ldots \bar{\vartheta}_{K, 1}^{j_{K, 1}} \ldots \bar{\vartheta}_{K, m_{K}}^{j_{K, m_{K}}}
$$

такие, что

$$
j_{k, 1}+\ldots+j_{k, m_{k}}=d_{k}, \quad j_{k, r} \geqslant 0, \quad r=1, \ldots, m_{k}, \quad 0 \leqslant d_{k}<M_{k}, \quad k=1, \ldots, K,
$$

попарно различны. Докажем это. Ввиду (61)

$$
\begin{aligned}
\bar{\vartheta}_{1,1}^{j_{1,1}} \ldots \bar{\vartheta}_{1, m_{1}}^{j_{1, m_{1}}} \ldots \bar{\vartheta}_{K, 1}^{j_{K, 1}} \ldots & \bar{\vartheta}_{K, m_{K}}^{j_{K, m_{K}}} \\
& =\bar{\vartheta}_{1}^{j_{1,1}+q j_{1,2}+\ldots+q^{m_{1}-1} j_{1, m_{1}}} \ldots \bar{\vartheta}_{K}^{j_{K, 1}+q j_{K, 2}+\ldots+q^{m_{K}-1} j_{K, m_{K}}} .
\end{aligned}
$$

Введем обозначения

$$
b_{k}=j_{k, 1}+q j_{k, 2}+\ldots+q^{m_{k}-1} j_{k, m_{k}}, k=1, \ldots, K
$$

Тогда множество элементов (63) запишется следующим образом:

$$
\bar{\vartheta}_{1}^{b_{1}} \ldots \bar{\vartheta}_{K}^{b_{K}}, \quad b_{k}=0,1, \ldots, q^{m_{k}}-1, \quad 0 \leqslant w_{q}\left(b_{k}\right)<M_{k}, \quad k=1, \ldots, K .
$$

Предположим, что два таких элемента совпадают:

$$
\begin{aligned}
\bar{\vartheta}_{1}^{b_{1}} \ldots \bar{\vartheta}_{K}^{b_{K}} & =\bar{\vartheta}_{1}^{b_{1}^{\prime}} \ldots \bar{\vartheta}_{K}^{b_{K}^{\prime}} \\
b_{k}, b_{k}^{\prime} & =0,1, \ldots, q^{m_{k}}-1, \quad 0 \leqslant w_{q}\left(b_{k}\right), w_{q}\left(b_{k}^{\prime}\right)<M_{k}, \quad k=1, \ldots, K .
\end{aligned}
$$

Так как $\vartheta_{k}$ - корень многочлена Галуа $F_{k}(x)$ степени $m_{k}$, то $\bar{\vartheta}_{k} \in \operatorname{GF}\left(q^{m_{k}}\right), k=$ $1, \ldots, K$. Поскольку числа $m_{1}, \ldots, m_{K}$ попарно взаимно просты, из равенства (64) следует, что

$$
\bar{\vartheta}_{k}^{b_{k}-b_{k}^{\prime}} \in \bar{R}=\mathrm{GF}(q), \quad k=1, \ldots, K .
$$

Так как $\bar{F}_{k}(x)$ - многочлен максимального периода, то $\bar{\vartheta}_{k}$ - примитивный элемент поля $\mathrm{GF}\left(q^{m_{k}}\right), k=1, \ldots, K$. В силу следующей леммы $b_{k}=b_{k}^{\prime}, k=1, \ldots, K$, что завершает доказательство предложения 14.

Лемма 2. Если $\vartheta-$ примитивный элемент поля $\operatorname{GF}\left(q^{m}\right) u \vartheta^{a-b} \in \operatorname{GF}(q)$, әде

$$
a, b \in\left\{0,1, \ldots, q^{m}-1\right\}, \quad 0 \leqslant w_{q}(a), w_{q}(b)<\min \left\{m, \frac{q-2}{q-1} m+1\right\}
$$

mo $a=b$. 
Доказательство получается из доказательства леммы 1 из [7] заменой $p$ на $q$. Предложение 15. Пусть в условиях предложения 14

$$
u_{k} \in L_{R}\left(F_{k}\right), \quad \bar{u}_{k} \neq 0, \quad k=1, \ldots, K, \quad a \in R, \quad 0 \leqslant d \leqslant q-1 .
$$

Тогда многочлен $F^{\left(d_{1}, \ldots, d_{K}, \lambda(a)\right)}(x)$ является минимальным многочленом ЛРП $w=a u_{1}^{d_{1}} \ldots u_{K}^{d_{K}} u \operatorname{rank} w=\left|J_{p}(\mathbf{d}, \mathbf{m}, \lambda(a))\right|$.

Доказательство. Так как выполнены условия предложения 10, справедливо соотношение (54). Поскольку $\bar{u}_{k} \neq 0$, в биномиальном представлении (52) $\bar{c}_{k r} \neq 0$, $r=1, \ldots, m_{k}, k=1, \ldots, K$. Тогда из доказательства предложения 10 следует, что коэффициент при каждой биномиальной последовательности в сумме (54) не равен нулю. Поэтому минимальный многочлен последовательности, являющейся слагаемым в (54), равен

$$
x-\vartheta_{1,1}^{j_{1,1}} \ldots \vartheta_{1, m_{1}}^{j_{1, m_{1}}} \ldots \vartheta_{K, 1}^{j_{K, 1}} \ldots \vartheta_{K, m_{K}}^{j_{K, m_{K}}} .
$$

Как показано выше, все элементы (63) попарно различны. Следовательно, ввиду второго утверждения предложения 3 , указанные минимальные многочлены попарно взаимно просты. Теперь из (54) и предложения 12 получаем, что $F^{\left(d_{1}, \ldots, d_{K}, \lambda(a)\right)}(x)$ - минимальный многочлен ЛРП $w$, и $\operatorname{rank} w=\left|J_{p}(\mathbf{d}, \mathbf{m}, \lambda(a))\right|$.

Теорема 8. Пусть $R$ - конечное локальное хольцо, $F_{1}(x), \ldots, F_{K}(x)$ - многочлены Галуа над $R$ степеней $m_{1}, \ldots, m_{K} \geqslant 1$ тахие, что $\bar{F}_{1}(x), \ldots, \bar{F}_{K}(x)$ - многоилены махсимального периода над полем $\bar{R}, u_{k} \in L_{R}\left(F_{k}\right), \bar{u}_{k} \neq 0, k=1, \ldots, K$, $\Phi\left(x_{1}, \ldots, x_{K}\right)$ - многочлен над колъцом $R$. Предположсим, что числа $m_{1}, \ldots, m_{K}$ попарно взаимно просты и степень многочлена $\Phi$ по переменной $x_{k}$ меньие $M_{k}$, $k=1, \ldots, K$.

Тогда многочлен $F^{(\Phi)}(x)$, определенный в (55), является минималъным многоиленом ЛРП $v=\Phi\left(u_{1}, \ldots, u_{K}\right)$ и оченки (56), (57) обращаяотсл в равенство.

Дохазательство. По условию многочлен $\Phi$ представляется в виде

$$
\Phi\left(x_{1}, \ldots, x_{K}\right)=\sum_{0 \leqslant d_{k}<M_{k}, k=1, \ldots, K} \varphi_{d_{1} \ldots d_{K}} x_{1}^{d_{1}} \ldots x_{K}^{d_{K}}
$$

Тогда

$$
v=\sum_{0 \leqslant d_{k}<M_{k}, k=1, \ldots, K} \varphi_{d_{1} \ldots d_{K}} u_{1}^{d_{1}} \ldots u_{K}^{d_{K}} .
$$

По предложению 15 многочлен $F^{\left(d_{1}, \ldots, d_{K}, \lambda\left(\varphi_{d_{1} \ldots d_{K}}\right)\right)}(x)$ является минимальным многочленом ЛРП $\varphi_{d_{1} \ldots d_{K}} u_{1}^{d_{1}} \ldots u_{K}^{d_{K}}$ при $0 \leqslant d_{k}<M_{k}, k=1, \ldots, K$. Из (50) и предложения 14 следует, что указанные многочлены попарно взаимно просты. Согласно предложению 12 произведение этих многочленов, то есть многочлен $F^{(\Phi)}(x)$, является минимальным многочленом ЛРП $v$.

Следствие 9. В условиях теоремъ 8 пусть

$$
D=\left\{\left(d_{1}, \ldots, d_{K}\right) \in \mathbf{N}_{0}^{K}: \varphi_{d_{1} \ldots d_{K}} \neq 0\right\} .
$$


Tогда

$$
\begin{aligned}
\sum_{\left(d_{1}, \ldots, d_{K}\right) \in D} \prod_{k=1}^{K}\left(\begin{array}{c}
m_{k}+\nu_{0}\left(d_{k}\right)-1 \\
\nu_{0}\left(d_{k}\right)
\end{array}\right)\left(\begin{array}{c}
m_{k}+\nu_{1}\left(d_{k}\right)-1 \\
\nu_{1}\left(d_{k}\right)
\end{array}\right) \ldots \leqslant \operatorname{rank} v \\
\leqslant \sum_{\left(d_{1}, \ldots, d_{K}\right) \in D} \prod_{k=1}^{K}\left(\begin{array}{c}
m_{k}+d_{k}-1 \\
d_{k}
\end{array}\right) .
\end{aligned}
$$

Доказательство. Ввиду (50)

$$
\prod_{\left(d_{1}, \ldots, d_{K}\right) \in D} F^{\left(d_{1}, \ldots, d_{K}, 0\right)}(x)\left|F^{(\Phi)}(x), \quad F^{(\Phi)}(x)\right| \prod_{\left(d_{1}, \ldots, d_{K}\right) \in D} F^{\left(d_{1}, \ldots, d_{K}\right)}(x) .
$$

При этом справедливо равенство (62), а так как элементы (63) попарно различны, то и неравенство (51) обращается в равенство. Отсюда следуют требуемые оценки (отметим, что верхняя оценка уже была доказана ранее, см. (30)).

Следствие 10. Если в условиях теоремы 8 степень многочена $\Phi$ по каждой переменной не превосходит $p-1$, то

$$
\begin{aligned}
F^{(\Phi)}(x) & =\prod_{\left(d_{1}, \ldots, d_{K}\right) \in D} F^{\left(d_{1}, \ldots, d_{K}\right)}(x), \\
\operatorname{rank} v & =\sum_{\left(d_{1}, \ldots, d_{K}\right) \in D}\left(\begin{array}{c}
m_{1}+d_{1}-1 \\
d_{1}
\end{array}\right) \ldots\left(\begin{array}{c}
m_{K}+d_{K}-1 \\
d_{K}
\end{array}\right),
\end{aligned}
$$

то есть для ранга достигается оченка (30).

Эти утверждения следуют из теоремы 8 и соотношений (49), (62).

Следствие 11. Пусть в условиях теоремъ $8 R$ - кольцо главных идеалов с радихалом $J(R)=\pi R$ индехса нильпотентности $n$. Тогда

$$
\operatorname{Ann}(v)=\left(F^{(\Phi)}(x), \pi F^{(\pi \Phi)}(x), \ldots, \pi^{n-1} F^{\left(\pi^{n-1} \Phi\right)}(x)\right) .
$$

Доказательство повторяет доказательство следствия 8.

Замечание 3. Для последовательностей над конечным полем в случае, когда многочлен $\Phi\left(x_{1}, \ldots, x_{K}\right)$ свободен от квадратов, утверждение следствия 10 доказано в [22]. Если многочлен $\Phi$ свободен от квадратов, то для него выполняются условия теоремы 8 (при $m_{k} \geqslant 2, k=1, \ldots, K$ ). Поэтому теорема 8 и следствие 10 обобщают результат статьи [22] не только на последовательности над кольцом $R$, но и на более широкий класс многочленов $\Phi$. Отметим, однако, что в случае, когда многочлен $\Phi$ свободен от квадратов, результат следствия 10 доказан в $[14,15,16,22]$ при значительно более общих условиях на последовательности $u_{1}, \ldots, u_{K}$ над конечным полем. Таким образом, по сравнению с указанными статьями, в теореме 8 (если ее рассматривать в случае, когда $R$ - конечное поле) рассматриваются многочлены $\Phi$ более общего вида, но на последовательности $u_{1}, \ldots, u_{K}$ накладываются более сильные ограничения.

Замечание 4. Результаты этого параграфа сохраняются в случае, когда $R$ - локальное кольцо с нильпотентным радикалом и конечным полем вычетов. Пример бесконечного кольца такого вида приведен в замечании 1. 


\section{Список литературы}

1. Атья М., Макдональд И., Введение в коммутативнуяо алгебру. Мир, Москва, 1972.

2. Бурбаки Н., Коммутативная алгебра. Мир, Москва, 1971.

3. Ван дер Варден Б. Л., Алгебра. Наука, Москва, 1979.

4. Глухов М. М., Елизаров В. П., Нечаев А. А., Алгебра. Часть II. Москва, 1991.

5. Зарисский О., Самюэль П., Коммутативяая алгебра. ИЛ, Москва, 1963.

6. Куракин В. Л., Представления над кольцом $\mathbf{Z}_{p^{n}}$ линейной рекуррентной последовательности максимального периода над полем GF(p). Дискретная математика (1992) 4, № 4, 96-116.

7. Куракин В. Л., Полиномиальные преобразования линейных рекуррентных последовательностей над кольцом $\mathrm{Z}_{p^{2}}$. Дискретнал математика (1999) 11, № 2, 40-65.

8. Лидл Р., Нидеррайтер Г., Конечные поля. т. 1, 2. Мир, Москва, 1988.

9. Нечаев А. А., Линейные рекуррентные последовательности над коммутативными кольцами. Дискретная математиха (1991) 3, № 4, 107-121.

10. Сачков В. Н., Введение в комбинаторяве методы дискретной математики. Наука, Москва, 1982.

11. Bernasconi J., Günter C. G., Analysis of a nonlinear feedforward logic for binary sequence generators. Lect. Notes Comput. Sci. (1986) 219, 161-166.

12. Brynielsson L., On the linear complexity of combined shift register sequences. Lect. Notes Comput. Sci. (1986) 219, 156-160.

13. Chan A. H., Goresky M., Klapper A., On the linear complexity of feedback registers. IEEE Trans. Inform. Theory, (1990) 36, № 3, 640-644.

14. Golić S. D., On the linear complexity of functions of periodic GF $(q)$ sequences. IEEE Trans. Inform. Theory (1989) 35, № 1, 69-75.

15. Herlestam $\mathrm{T}$., On the complexity of functions of linear shift register sequences. Int. Symp. Inform. Theory, Les Arc, France, 1982.

16. Herlestam T., On functions of linear shift register sequences. Lect. Notes Comput. Sci. (1986) 219 (1986), 119-129.

17. Key E. L., An analysis of the structure and complexity of nonlinear binary sequence generators. IEEE Trans. Inform. Theory (1976) 22, № 6, 732-736.

18. Klapper A., The vulnerability of geometric sequences based on fields of odd characteristic. J. Cryptology, (1994) 7, 33-51.

19. Kurakin V. L., Kuzmin A. S., Mikhalev A. V., Nechaev A. A., Linear recurring sequences over rings and modules. J. Math. Sci. (1995) 76, № 6, 2793-2915.

20. Lu P., Song G., Feasible calculation of the generator for combined LFSR sequences. Lect. Notes Comput. Sci. (1991) 508, 86-95.

21. Lu P., Song G., Zhou J., Tenzor product with application to linear recurring sequences. $J$. Math. Res. Exposition (1992) 12, № 4, 551-558.

22. Rueppel R. A., Staffelbach O. J., Products of linear recurring sequences with maximum complexity. IEEE Trans. Inform. Theory (1987) 33, № 1, 126-131.

23. Selmer E. S., Linear Recurrence Relations Over Finite Fields. Univ. Bergen, Bergen, 1966.

24. Vajda I., Nemetz T., Substitution of characters in $q$-ary $m$-sequences. Lect. Notes Comput. Sci. (1991) 508, 96-105.

25. Zierler N., Mills W. H., Products of linear recurring sequences. J. Algebra (1973) 27, № 1, 147-157. 